FPAUO-12/08

\title{
Non-Abelian T-duality and consistent truncations in type-II supergravity
}

\author{
Georgios Itsios $^{1,3 a}$ ，Yolanda Lozano ${ }^{2 b}$, \\ Eoin Ó Colgáin ${ }^{2 c}$ and Konstadinos Sfetsos ${ }^{1 d}$ \\ ${ }^{1}$ Department of Engineering Sciences, University of Patras, \\ 26110 Patras, Greece \\ ${ }^{2}$ Department of Physics, University of Oviedo, \\ Avda. Calvo Sotelo 18, 33007 Oviedo, Spain \\ ${ }^{3}$ Centro de Fisica do Porto \& Departamento de Fisica e Astronomia, \\ Faculdade de Ciencias da Universidade do Porto, Rua do Campo Alegre 687, \\ 4169-007 Porto, Portugal
}

\begin{abstract}
For a general class of $S O(4)$ symmetric backgrounds in type-II supergravity, we show that the action of non-Abelian T-duality can be described via consistent truncation to seven dimensional theories with seemingly massive modes. As such, any solution to these theories uplifts to both massive type IIA and IIB supergravities presenting an invertible map between the two. For supersymmetric backgrounds, we show that for spinors transforming under $S O(4)$ non-Abelian T-duality breaks the original supersymmetry by half. We use these mappings to generate the non-Abelian T-duals of the maximally supersymmetric pp-wave, the Lin, Lunin, Maldacena geometries and spacetimes with Lifshitz symmetry.
\end{abstract}

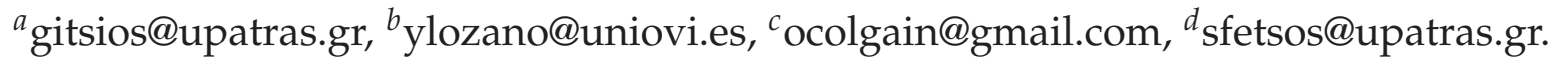




\section{Contents}

1 Introduction

2 Consistent KK Reduction 4

2.1 KK reduction on $s^{3} \ldots \ldots \ldots \ldots \ldots$

2.2 Non-Abelian T-duality and KK reduction . . . . . . . . . . . . . 8

2.3 Reduction at the level of the action . . . . . . . . . . . . 11

\begin{tabular}{llr}
3 & Supersymmetry & 12 \\
\hline
\end{tabular}

4 Massive IIA non-Abelian T-duals 18

5 Explicit examples $\quad 19$

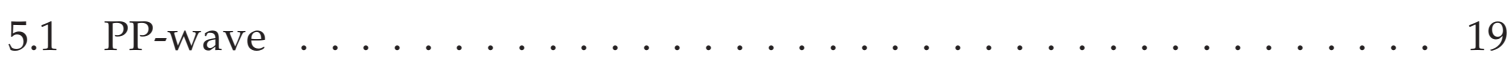

5.2 Type-IIB backgrounds with $S O(4) \times S O(4) \times \mathbb{R}$ isometry . . . . . . 21

5.3 Lifshitz symmetry solutions . . . . . . . . . . . . . . . 23

6 Concluding remarks $\quad 25$

A Review of type-II supergravities 27

A.1 Type-IIB supergravity . . . . . . . . . . . . . . . 27

A.2 Massive IIA supergravity . . . . . . . . . . . . . . . 28

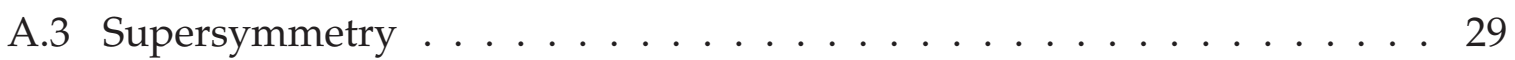

B Details of various KK reductions

B.1 Reduction of type-IIB on $S^{3} \ldots \ldots \ldots \ldots$

B.2 Reduction of massive IIA on the non-Abelian T-dual . . . . . . . . . . 32

B.3 Reduction of massive IIA on $S^{3} \ldots \ldots \ldots \ldots$

C Details of derivation of the T-dual Ramond fields 34

C.1 Type-IIB to massive IIA . . . . . . . . . . . . . . . . 35

C.2 Massive IIA to IIB . . . . . . . . . . . . . . . . . . . 36 


\section{Introduction}

Recently our understanding of non-Abelian T-duality [1, 2, 3] has been considerably advanced by showing how to implement the duality transformation on solutions of type-IIA (massive) and type-IIB supergravities with non-trivial RR fluxes and a nonAbelian group of isometries [4]. Originally the formulation was implemented on supergravity backgrounds in which isometries were realized with group spaces [4]. Nevertheless, it was soon extended to cover backgrounds in which the isometries were realized with coset spaces [5] as in the vast majority of interesting solutions appearing in supergravity and in string theory.

In the present paper we put on firmer ground the previous work by focusing not on particular supergravity solutions but on the corresponding massive IIA and type-IIB supergravity theories themselves. As a playground we choose a general class of $S O(4)$ symmetric backgrounds and examine non-Abelian T-duality with respect to an $S U(2)$ subgroup 1 We unearth consistent reduction ansatze to the same underlying seven dimensional theories meaning that any solution to these lower-dimensional actions uplifts simultaneously to a solution of both type-IIA and type-IIB supergravities. Some of our motivation lends itself to the important work of Bergshoeff, Hull \& Ortín [6] where the Abelian T-duality rules are derived via simultaneous circle reductions and a subsequent matching of the fields using the fact that the $\mathcal{N}=2$ nine-dimensional supergravity is unique.

Despite the casual analogy, in venturing from circle reductions to geometries connected via non-Abelian T-duality, one has an important obstacle to clear; non-Abelian T-duality breaks isometries, and in the particular setting of this paper, one has to compare an $S^{3}$ reduction of the original geometry with an $\mathbb{R} \times S^{2}$ reduction in the T-dual. Indeed, as we shall see, delicate cancellations have to happen where the Einstein equations along $S^{3}$ get mapped to those along $\mathbb{R} \times S^{2}$ and the $B$-field equation in the T-dual conspires to mimic this result. Another potential surprise may be that the non-Abelian transformation of the original RR flux ansatz leads one to a T-dual reduction ansatz that reproduces the same lower-dimensional theory. A priori, it is not obvious that some reshuffling of the field content may not be required. However, this theory, which arises as an $S^{3}$ reduction from type-IIB with $S O(4)$ singlets retained, does not agree with the Lagrangian expected from a (warped) $S^{3}$ reduction from type-IIB [7], since

\footnotetext{
${ }^{1}$ Neglecting a $B$-field with field strength $H$ along $S^{3}$, this is the most general ansatz.
} 
although some fields, such as the dilaton, fit into the massless supergravity multiplet and conform to expectations, the warp factor and the axion, the other $S O(4)$-singlet scalars of the reduction, appear to be massive.

Recall that the identification of consistent dimensional reductions can be a conceptually and technically demanding task. Nevertheless, our most celebrated examples of consistent truncations involve maximally symmetric sphere reductions [8]-[14] where there are no obvious guidelines to constructing a consistent reduction ansatz. In contrast, consistent truncations are relatively easy to work out when one has generic $S U(3)$-structure [15]-[19] or $S U(2)$-structure [20]-[24] manifolds allowing the possibility to expand in the invariant forms 2 In addition, new reduction ansatze may sometimes be deduced from known ansatze, as in [26], where an $S^{3}$ reduction in type IIA is derived as a limit of the $S^{4}$ reduction of [13] involving the $S^{4}$ pinching off to $\mathbb{R} \times S^{3}$, or alternatively via Abelian T-duality [27]. In this paper, the underlying principle illuminating the reduction on the T-dual spacetime with factor $\mathbb{R} \times S^{2}$ is non-Abelian T-duality.

Non-Abelian T-duality breaks isometries, and as such, it is expected that supersymmetry is also broken 3 From our extensive knowledge of Abelian T-duality in type-II supergravity, we know that the Killing spinor equations of the original solution can be mapped to the Killing spinor equations of the T-dual via a rotation acting exclusively on one of the chiral Killing spinors, and that in the process, the chirality of the spinor flips [29]. Here we generalise this by showing that there is an analogous rotation when we perform an $S U(2)$ transformation and that the Killing spinor equations of the Tdual are those of the original up to the imposition of a single further supersymmetry variation. For spinors transforming under $S O(4)$ we show that this additional condition is consistent with half of the supersymmetries, those corresponding to the $S U(2)$ isometry with respect to which we perform the non-Abelian T-duality, breaking.

The organization of the paper is as follows: In section 2 we present the general class of type-II $S O(4)$ symmetric backgrounds that we T-dualize with respect to an SU(2) subgroup. Focusing on type-IIB we reduce both the original IIB background (on $S^{3}$ ) and its massive type-IIA dual (on $\mathbb{R} \times S^{2}$ ) and obtain a consistent truncation in seven dimensions, both at the level of the equations of motion and at the level of the actions.

\footnotetext{
${ }^{2}$ For the reduction of fermions see [25].

${ }^{3}$ This is correct at the supergravity level. In string theory supersymmetry can be realized in a nonlocal way (see [28] and references therein).
} 
We compare the resulting seven dimensional action to previous constructions of maximal supergravities in the literature. In section 3 we analyze the supersymmetry of the non-Abelian dual. We show that the mapping of the Killing spinor equations requires an additional condition, breaking the original supersymmetry by a half for spinors transforming under $S O(4)$. In section 4 we focus on massive type-IIA $S O(4)$ symmetric backgrounds and show that the reduction on $S^{3}$ produces as well a consistent truncation which is however different from the one obtained in the reduction of type-IIB on $S^{3}$. In section 5 we present three examples in type-IIB in which our nonAbelian T-duality transformation can be used to generate new solutions of massive IIA. These are the maximally supersymmetric pp-wave background [30], the general class of $1 / 2$ supersymmetric type-IIB solutions constructed in [31] and the Lifshitz solutions of [32]. We also exhibit the reverse transformation from type-IIA to typeIIB using the pp-wave. The non-Abelian dual of mass deformed ABJM provides an additional example in type-IIB very similar in nature to the dual of the $1 / 2$ supersymmetric solutions in [31]. Section 6 contains our conclusions and further directions. Appendix A summarizes some aspects of type-II supergravities relevant to our work. Appendix B contains the details of the reductions of type-IIB on $S^{3}$, type-IIA on $\mathbb{R} \times S^{2}$ and type-IIA on $S^{3}$. Finally, Appendix $C$ contains the details of the derivation of the dual Ramond fields, which is used as an ansatz in section 2.

\section{Consistent KK Reduction}

In this section, for concreteness, we confine ourselves to spacetimes with warped $S^{3}$ factors permitting a non-Abelian $S U(2)$ transformation. From a technical point of view since we will be dealing with a group manifold the isometry acts with no fixed points, a fact that, as in [4], facilitates the computations. However, we expect that the picture we paint here will generalise to the examples presented in [5] where one encounters larger isometry groups based on coset spaces, such as spheres, and the corresponding T-duals have less supersymmetry and fewer isometries.

As stated, we are interested in spacetimes of the form

$$
d s^{2}=d s^{2}\left(M_{7}\right)+e^{2 A} d s^{2}\left(S^{3}\right)
$$

where $M_{7}$ is a seven-dimensional Minkowskian spacetime and the warp factor $A$ de- 
pends only on the coordinates on $M_{7}$. The $S^{3}$ metric is normalized so that $R_{i j}=\frac{1}{2} g_{i j}$. The above metric has an $\mathrm{SO}(4)$ group of isometries, together with the isometries of the $M_{7}$ manifold in which we will not be interested in our general discussion. The NS-sector fields are comprised also by a two-form $B$ with field strength $H=d B$ with no-components along $S^{3}$, as well as a dilaton $\Phi$ which may depend on the coordinates of $M_{7}$. Consequently, these fields are also invariant under the $S O(4)$ isometry group. Incorporating a $B$-field along $S^{3}$ will lead to a generalisation which falls outside our scope in this paper. Irrespective of the chirality of the theory, one can write the $S O(4)$ as $S U(2)_{L} \times S U(2)_{R}$ and perform an $S U(2)$ transformation with respect to one of these factors as explained in detail in [4]. The end result is a spacetime with fields in the NS-sector given by

$$
\begin{aligned}
& d \hat{s}^{2}=d s^{2}\left(M_{7}\right)+e^{-2 A} d r^{2}+\frac{r^{2} e^{2 A}}{r^{2}+e^{4 A}} d s^{2}\left(S^{2}\right), \\
& \hat{B}=B+\tilde{B}, \quad \tilde{B}=\frac{r^{3}}{r^{2}+e^{4 A}} \operatorname{Vol}\left(S^{2}\right), \\
& e^{-2 \hat{\Phi}}=e^{-2 \Phi} e^{2 A}\left(r^{2}+e^{4 A}\right),
\end{aligned}
$$

where we have used hat notation to differentiate the T-dual fields from the original ones. Observe also that the $S U(2)$ isometry left untouched by the transformation is captured in the symmetries of the resulting $S^{2}$. The field strength, $\hat{H}=d \hat{B}$ may be written as

$$
\hat{H}=H+\left[\frac{r^{2}\left(r^{2}+3 e^{4 A}\right)}{\left(r^{2}+e^{4 A}\right)^{2}} d r-4 \frac{r^{3} e^{4 A}}{\left(r^{2}+e^{4 A}\right)^{2}} d A\right] \wedge \operatorname{Vol}\left(S^{2}\right) .
$$

To construct solutions of type-II supergravity, we need to complement our original spacetime ansatz (2.1), with knowledge of the RR fields. Building on the tradition started in [4], and the two known examples which will serve as valuable consistency checks, we begin by considering first type-IIB supergravity. Through the existence of the known examples, we know that our SU(2) transformation takes solutions of the equations of motion in type-IIB to solutions in massive IIA, strongly suggesting that by examining the equations of motion one can unearth some deeper structure. Indeed, as we shall see shortly, this is the case and the underlying structure that emerges is a unifying gravity description in seven dimensions via parallel consistent truncations on the original spacetime (2.1) and on the T-dual spacetime (2.2). As we shall see, in this formulation non-Abelian T-duality is invertible unlike the case of the standard 


\subsection{KK reduction on $S^{3}$}

Our first task is to identify the reduced seven dimensional theory. To do this we incorporate into our type-IIB ansatz the following RR fluxes that respect the symmetry of the round $S^{3}$ appearing in the metric

$$
\begin{aligned}
& F_{5}=G_{2} \wedge \operatorname{Vol}\left(S^{3}\right)-e^{-3 A} \star_{7} G_{2}, \\
& F_{3}=G_{3}-m \operatorname{Vol}\left(S^{3}\right), \\
& F_{1}=G_{1} .
\end{aligned}
$$

Note that the self-duality of the five-form has already been imposed according to our conventions, see (2.14) below. We take the forms $G_{i}, i=1,2,3$ to live on $M_{7}$. Thanks to the type-IIB Bianchi identities (see Appendix A.1) the parameter $m$ is a constant and as it turns out, it will be mapped to the mass parameter of the massive IIA supergravity. Aspects of type-II supergravities relevant to this work are reviewed in Appendix A.

The consistency of this reduction should come as no surprise and we have indeed checked that one can get the same result by performing the reduction at the level of the action. We enclose details of the type-IIB reduction in the Appendix B.1. In particular, the reduction implies that the forms we used in our ansatz can be expressed in terms of some potentials as

$$
G_{1}=d C_{0}, \quad G_{2}=d C_{1}-m B, \quad G_{3}=d C_{2}-C_{0} H .
$$

Hence the field content arising from the RR sector is a scalar, $C_{0}$, a one-form, $C_{1}$ and a two-form, $C_{2}$. These supplement the metric, the two-form $B$-field and the dilaton $\Phi$ from the NS sector. All these arise from the effective action which is given in the Einstein frame by (B.8). After performing the transformation

$$
\Phi=5 \tilde{\Phi}+\frac{3}{2} A,
$$

which allows us to diagonalize the action and compare dilaton factors directly with

\footnotetext{
${ }^{4}$ The transformation is invertible also in the context of Poisson-Lie T-duality in which a nonstandard $\sigma$-model action lacking manifest Lorentz invariance is used [33].
} 
the action (6.20) in [7], we arrive, dropping the tildes, at the final action in Einstein frame from whence all equations of motion may be derived. The corresponding Lagrangian density is

$$
\begin{aligned}
\mathcal{L}_{\text {Einstein }}= & R-3(\partial A)^{2}-20(\partial \Phi)^{2}-\frac{1}{2} e^{10 \Phi+3 A}\left(\partial C_{0}\right)^{2}-\frac{1}{12} e^{-8 \Phi} H^{2} \\
& -\frac{1}{2}\left(m^{2} e^{14 \Phi-3 A}-3 e^{4 \Phi-2 A}+\frac{1}{2} e^{6 \Phi-3 A} G_{2}^{2}+\frac{1}{6} e^{2 \Phi+3 A} G_{3}^{2}\right) \\
& +G_{2} \wedge C_{2} \wedge H,
\end{aligned}
$$

where we have made use of (2.5) and have replaced $G_{1}$ by $d C_{0}$.

Now that we have the seven-dimensional action in Einstein frame we can attempt to make contact with the supergravity literature. The warped $S^{3}$ reduction ansatz from type-IIB is still unknown, but various reductions from type-I supergravity have been discussed, notably the reduction ansatz of [34], which through the ten-dimensional equations of motion, reproduces the equations of motion of [35], and the ansatz of [36] which leads to [37]. More generally, in seven dimensions one can construct maximal supergravities [7] (see section 6.3) generalising [36, 37]. Attempts to match our action to the general action of [7] reveal that only the dilaton factors agree perfectly while neither our warp factor, $A$, nor the axion, $C_{0}$, fit into this work.

The expectation then is that $A$ and $C_{0}$ correspond to scalars in massive multiplets. As our potential has no stationary points, determining the mass of these terms relative to the dilaton becomes tricky. In spite of these difficulties, the supergravity spectrum for warped $S^{3}$ solutions corresponding to the near-horizon geometry of D5-branes may be found in table IV of [38]. One observes that in the full spectrum there are three $S O(4)$ singlet scalars, i.e. representation [00](000), which show up in three different multiplets: $n=2, n=3$ and $n=4$. Now only the $n=2$ multiplet corresponding to the dilaton is in the massless supergravity multiplet, while the other two are massive 5 This suggests that more generally $A$ and $C_{0}$ are massive modes and that their omission from the maximal supergravity action should not come as a surprise.

We also note that the overall coupling constant of the seven-dimensional theory in terms of the coupling constant of type-IIB supergravity is

$$
\frac{1}{2 \kappa_{7}^{2}}=\frac{\operatorname{Vol}\left(S^{3}\right)}{2 \kappa^{2}} .
$$

\footnotetext{
${ }^{5}$ We are grateful to H. Samtleben for correspondence on this point.
} 
In addition it is a lengthy but otherwise straightforward procedure to demonstrate that by dimensionally reducing the type-IIB supergravity action (A.1), then passing to the Einstein frame and finally by redefining as in (2.6) we obtain precisely the action (2.7). We have decided not to include the details of this calculation as it presented no technical or conceptual challenges.

\subsection{Non-Abelian T-duality and KK reduction}

We have consistently reduced the general $S O(4)$ invariant ansatz of type-IIB down to seven dimensions at the level of the equations of motion and noted that this is also possible at the action level. The question we would like to address now is whether such a reduction will be possible for the non-Abelian T-dual background with respect to an $S U(2) \subset S O(4)$. Indeed, our knowledge of non-Abelian T-duality in this setting is confined to two solitary examples constructed in [4] involving the near horizon geometry of the D1-D5- and the D3-brane systems corresponding to the $A d S_{3} \times S^{3} \times$ $T^{4}$ and the $A d S_{5} \times S^{5}$ geometries 6 So it may even be too much to expect that there is an overarching action in seven dimensions describing the full reduction and not just separate actions corresponding to a truncation to $m, G_{3}$ (D1-D5 near-horizon) and $A, G_{2}$ (D3 near-horizon) or $G_{1}$ separately. The surprise, as we shall see shortly, is that one obtains exactly the same theory in seven dimensions.

The form of the RR flux fields can certainly be constrained by the symmetries of the non-Abelian T-dual. It is apparent from the expressions for the NS sector (2.2) that in the non-Abelian T-dual backgrounds the $S O(4)$ isometry group is broken down to $S O(3) \sim S U(2)$, i.e. the symmetry group of $S^{2}$. Hence, we have two natural forms to build an RR flux reduction ansatz from, namely $d r$ and $\operatorname{Vol}\left(S^{2}\right)$. In type-IIA supergravity, decomposing the forms one has the natural ansatz

$$
\begin{aligned}
& \hat{F}_{2}=M_{0} \operatorname{Vol}\left(S^{2}\right)+M_{1} \wedge d r+M_{2}, \\
& \hat{F}_{4}=N_{1} \wedge d r \wedge \operatorname{Vol}\left(S^{2}\right)+N_{2} \wedge \operatorname{Vol}\left(S^{2}\right)+N_{3} \wedge d r+N_{4},
\end{aligned}
$$

where $M_{i}, N_{i}$ denote forms of degree $n$ living on $M_{7}$. The difficulty arises from the fact that all forms on $M_{7}$ in the above ansatz can still depend on the radial direction $r$. One approach then is to employ trial and error and match the equations of motion of mas-

\footnotetext{
${ }^{6}$ More examples were constructed in [5] involving coset and not group spaces.
} 
sive IIA supergravity to those of type-IIB, so that the $M_{i}$ and $N_{i}$ align with our $G_{i}$ from the type-IIB reduction discussed previously. While this approach may reap a reward if one just focuses on reproducing the equations of motion from type-IIB reduced on $S^{3}$, i.e. if one puts the answer in by hand, it is difficult to find a general reduction ansatz with cohomogeneity-one manifolds (for example, see [27]). Alternatively, from earlier work [4] it is known how the fluxes transform, so we can simply generate the appropriate ansatz using the type-IIB flux ansatz as a seed. Obviously this is the preferred approach which we follow.

Firstly, one constructs the type-IIB flux bispinor from the ansatz (2.4)

$$
P=\frac{e^{\Phi}}{2} \sum_{n=0}^{4} \frac{\not_{2 n+1}}{(2 n+1) !},
$$

where we have employed the usual notation $\mathbb{F}_{p} \equiv F_{i_{1} \ldots i_{p}} \Gamma^{i_{1} \ldots i_{p}}$, and reads off the T-dual bispinor from the transformation

$$
\hat{P}=P \Omega^{-1},
$$

where $\Omega$ is the Lorentz transformation matrix acting on the spinors. It reads [4]

$$
\Omega=\Gamma_{11} \frac{e^{2 A} \Gamma_{789}+\mathbf{x} \cdot \boldsymbol{\Gamma}}{\sqrt{r^{2}+e^{4 A}}} \quad \Longrightarrow \quad \Omega^{-1}=\Gamma_{11} \frac{e^{2 A} \Gamma_{789}-\mathbf{x} \cdot \boldsymbol{\Gamma}}{\sqrt{r^{2}+e^{4 A}}} .
$$

Note that we are using $e^{i}, i=7,8,9$ to denote the tangent space along the transformed T-dual space. A natural choice of frame may also be found in [4]

$$
\hat{e}^{i}=\frac{1}{\sqrt{r^{2}+e^{4 A}}}\left(e^{A} d x^{i}+x^{i} e^{-A} b(r) d r\right), \quad b(r)=\frac{\sqrt{r^{2}+e^{4 A}}-e^{2 A}}{r} .
$$

Our conventions on Hodge duality are such that on a $p$-form in a $D$-dimensional spacetime

$$
\left(\star F_{p}\right)_{\mu_{p+1} \cdots \mu_{D}}=\frac{1}{p !} \sqrt{|g|} \epsilon_{\mu_{1} \cdots \mu_{D}} F_{p}^{\mu_{1} \cdots \mu_{p}},
$$

where $\epsilon_{01 \ldots 9}=1$. With this we have the useful identity $\star \star F_{p}=s(-1)^{p(D-p)} F_{p}$, where $s$ is the signature of spacetime which we take to be mostly plus. In our case, the indices, $\mu=0,1, \ldots, 6$ refer to the seven-dimensional spacetime $M_{7}$ of Minkowski signature, whereas 7, 8,9 either to the $S^{3}$ directions or, for the non-Abelian T-dual, to the frame defined in (2.13). 
The details of the construction are presented in Appendix C.1. The final form of the fluxes may then be read off from the T-dual bispinor

$$
\hat{P}=\frac{e^{\hat{\Phi}}}{2} \sum_{n=0}^{5} \frac{\hat{F}_{2 n}}{(2 n) !} .
$$

This procedure gives the massive IIA fluxes, that we read from equation (C.7)

$$
\begin{aligned}
& \hat{F}_{0}=m, \\
& \hat{F}_{2}=\frac{m r^{3}}{r^{2}+e^{4 A}} \operatorname{Vol}\left(S^{2}\right)+r d r \wedge G_{1}-G_{2}, \\
& \hat{F}_{4}=\frac{r^{2} e^{4 A}}{r^{2}+e^{4 A}} G_{1} \wedge d r \wedge \operatorname{Vol}\left(S^{2}\right)-\frac{r^{3}}{r^{2}+e^{4 A}} G_{2} \wedge \operatorname{Vol}\left(S^{2}\right)+r d r \wedge G_{3}+e^{3 A} \star_{7} G_{3} .
\end{aligned}
$$

A quick inspection shows that they are of the form (2.9). One can now use these fluxes in tandem with the T-dual spacetime (2.2) and plug them both into the massive IIA equations of motion. We spare the reader the details but just summarize the necessary steps. From the Bianchi identities and the flux equations of motion one recovers (B.1) and the last two eqs. of (B.2). From the B-field equation of motion one gets the first of (B.2) and

$$
\begin{gathered}
\frac{1}{2} e^{A-2 \Phi} \operatorname{Vol}\left(M_{7}\right)-d\left(e^{3 A-2 \Phi} \star_{7} d A\right)=\frac{1}{4}\left(m^{2} e^{-3 A}-e^{3 A} G_{1} \wedge \star_{7} G_{1}\right. \\
\left.+e^{-3 A} G_{2} \wedge \star_{7} G_{2}-e^{3 A} G_{3} \wedge \star_{7} G_{3}\right)
\end{gathered}
$$

Observe that (2.17) is just (B.3) written in a form notation. The Einstein equations in the $r$-direction and the directions along $S^{2}$ deliver (B.3). In all cases the dependence on the $r$-coordinate drops out. Finally, one recovers the seven-dimensional Einstein equation (B.4).

Now that we have discussed two consistent reduction ansatze from type-II leading to the same gravitational action in seven dimensions, we pause to recap on what we have shown. Notably, any solution to the action (2.7) may be uplifted simultaneously to both type-IIB and massive IIA.7 Therefore, in this formulation we have circumvented the problem of non-invertibility of non-Abelian T-duality in its treatment in

\footnotetext{
${ }^{7}$ Another prominent example of non-unique uplifts of solutions to higher dimensions includes solutions of the Romans theory in five dimensions [39]. These can be uplifted to type-IIB supergravity [9] and to eleven-dimensional supergravity [40,41]. However, unlike our case, there is no obvious relation between these distinct uplifts.
} 
the standard two-dimensional $\sigma$-model approach, namely the fact that it is not possible to reconstruct the original background from its non-Abelian dual due to its lack of isometries. In addition, starting with a type-IIB geometry with an $S^{3}$, non-Abelian Tduality will generate a background to massive type-IIA whenever the original $F_{3}$-flux extends on the $S^{3}$ directions (see eq. (2.4)). Therefore non-Abelian duality can be used as a principle to generate new solutions to massive type-IIA supergravity.

\subsection{Reduction at the level of the action}

It has already been pointed out that the seven-dimensional action (2.7), besides giving rise to the same equations of motion following either from the general $S O(4)$ invariant ansatz (2.1) and (2.4) or from its non-Abelian dual (2.2) and (2.16), also arises from the dimensional reduction of the type-IIB action on $S^{3}$. Conceptually, it is not obvious that a dimensional reduction of massive IIA on the T-dual background with topology $\mathbb{R} \times S^{2}$ will result into the same action (2.7), or at least, if that is the case, it should happen in a non-trivial way. Unlike the case of the usual dimensional reduction on compact manifolds where the zero mode in a harmonic expansion of the various fields is retained, the non-Abelian T-dual background seems to be non-compact due to the fact that the radial coordinate $r$ seems to take values in the entire half real line. In order to fully clarify the global topological properties of the dual background we should resort to the $\sigma$-model derivation. However, how to extract topological information in the non-Abelian case is not clear (see [42]). In any case in order to reproduce (2.7) it is key that the dual background has topology $\mathbb{R} \times S^{2}$, as we show below. Recall also that a treatment of non-Abelian T-duality of exact Conformal Field Theory models in [43] involving only NS fields, led to the conclusion that non-Abelian T-duals effectively capture states of some parent theory corresponding to group theory representations with infinitely high highest weight. Based on that work we expect that the dimensional reduction of non-Abelian duals will capture this phenomenon.

Keeping these in mind we start reducing the massive IIA action on the dual background. All relevant terms are given in appendix B.2. Substituting them into (A.11) and after a partial integration, one obtains 


$$
\begin{aligned}
S=\frac{\operatorname{Vol}\left(S^{2}\right)}{2 \kappa^{2}} \int & d r r^{2} \sqrt{-g}\left\{e ^ { 3 A - 2 \Phi } \left(R+6(\partial A)^{2}-12 \partial A \cdot \partial \Phi+4(\partial \Phi)^{2}\right.\right. \\
& \left.-\frac{H^{2}}{12}+\frac{e^{-2 A}}{2\left(r^{2}+e^{4 A}\right)^{2}}\left(3 r^{4}+6 r^{2} e^{4 A}+8 r^{2} e^{2 A}+27 e^{8 A}\right)\right) \\
& \left.-\frac{1}{2}\left(e^{-3 A} m^{2}+e^{3 A} G_{1}^{2}+\frac{e^{-3 A}}{2} G_{2}^{2}+\frac{e^{3 A}}{6} \frac{r^{2}-e^{4 A}}{r^{2}+e^{4 A}} G_{3}^{2}\right)\right\} \\
& -\frac{\operatorname{Vol}\left(S^{2}\right)}{2 \kappa^{2}} \int d r \frac{r^{4}}{r^{2}+e^{4 A}} C_{2} \wedge G_{2} \wedge H \wedge d r .
\end{aligned}
$$

In the above action, there are divergent integrals with respect to $r$. To deal with them we perform this integration from 0 to $R_{0}$, where $R_{0}$ is a cutoff which we take much larger than $e^{2 A}$ while keeping as well the dominant term. Then we can write the above action in the form (B.6) but with overall coefficient

$$
\frac{\operatorname{Vol}\left(S_{R_{0}}^{2}\right)}{2 \kappa^{2}}=\frac{1}{2 \kappa_{7}^{2}}
$$

where $\operatorname{Vol}\left(S_{R_{0}}^{2}\right)=\frac{4}{3} \pi R_{0}^{3}$ is the volume of this large 2-sphere. In order to keep the seven-dimensional Planck constant finite we have to take the ten dimensional coupling constant $\kappa^{2}$ very large as well so that the ratio is finite. This is in resonance with the results of [43] where the overall coupling constant of the theory had to be taken large in order to accommodate the consistent description of states corresponding to infinitely large highest weight group theory representations.

\section{Supersymmetry}

In this section we examine the supersymmetry of the massive IIA T-dual theory in relation to that of the original type-IIB theory. In particular, we are interested in uncovering the mapping of the corresponding Killing spinor equations. In addition, we are after a general statement concerning the amount of supersymmetry preserved under the non-Abelian T-duality transformation. Based on the examples of [4], where a key role was played by the Lie-Lorentz or Kosmann derivative on spinors [44, 45, 46], we expect that for spinors transforming under $S O(4)$ supersymmetry is reduced by half, a statement that we actually prove below in this section.

We will work with a consistent set of conventions [29] reproduced in Appendix A.3. 
It it instructive to consider first the non-Abelian T-dual of the flat spacetime metric

$$
d s^{2}=-d t^{2}+\cdots+d x_{5}^{2}+d x^{2}+\frac{x^{2}}{4} d s^{2}\left(S^{3}\right),
$$

without any RR fluxes, or other fields excited. Here the Killing spinor equation tells us that the spinor is covariantly constant, namely $D_{\mu} \epsilon=0$. As before the $S^{3}$ metric is normalized such that $R_{i j}=\frac{1}{2} g_{i j}$. We conveniently write the $S^{3}$-metric as

$$
d s^{2}\left(S^{3}\right)=4\left(d \theta^{2}+\cos ^{2} \theta d \phi^{2}+\sin ^{2} \theta d \psi^{2}\right) .
$$

In this coordinate system the Killing spinor equation is solved by

$$
\epsilon=\exp \left(\frac{\theta}{2} \Gamma^{x \theta}\right) \exp \left(\frac{\phi+\psi}{2} \Gamma^{x \phi}\right) \epsilon_{0}
$$

with $\epsilon_{0}$ being a constant spinor and where we have used the natural orthonormal frame suggested by the form of the metric. Indices in Gamma matrices belong to the tangent space. The non-Abelian T-dual background is found from (2.2) to be

$$
\begin{aligned}
d s^{2} & =-d t^{2}+\cdots+d x_{5}^{2}+d x^{2}+\frac{4}{x^{2}} d r^{2}+\frac{4 r^{2} x^{2}}{16 r^{2}+x^{4}} d s^{2}\left(S^{2}\right) \\
B & =\frac{16 r^{3}}{16 r^{2}+x^{4}} \operatorname{Vol}\left(S^{2}\right) \\
\Phi & =-\frac{1}{2} \ln \left[\frac{x^{2}}{4}\left(r^{2}+\frac{x^{4}}{16}\right)\right] .
\end{aligned}
$$

The dilatino variation in (A.15) or equivalently (A.16) is solved through the projector

$$
\Gamma^{x r \theta \phi} \sigma^{3} \epsilon=-\epsilon
$$

where $\theta$ and $\phi$ refer to the coordinates along $S^{2}$. The gravitino variation of the same equations can also be readily solved by

$$
\epsilon=\exp \left[-\frac{1}{2} \tan ^{-1}\left(\frac{1}{4} \frac{x^{2}}{r}\right) \Gamma^{\theta \phi} \sigma^{3}\right] \exp \left(\frac{\theta}{2} \Gamma^{x \theta}\right) \exp \left(\frac{\phi}{2} \Gamma^{\theta \phi}\right) \epsilon_{0},
$$

where $\epsilon_{0}$ denotes a constant spinor. We see that supersymmetry has been broken by one half through the introduction of the projection condition in (3.5).

The above expression for the Killing spinor, after reintroducing the original warped 
factor by replacing $\frac{x^{2}}{4}$ with $e^{2 A}$, suggests a substitution of the form

$$
\epsilon=e^{X} \tilde{\epsilon}=\exp \left(-\frac{1}{2} \tan ^{-1}\left(\frac{e^{2 A}}{r}\right) \Gamma^{\theta \phi} \sigma^{3}\right) \tilde{\epsilon},
$$

into the Killing spinor equations of the original theory either in type-IIB or in massive IIA. The above rotation is expected in the sense that a non-Abelian T-duality results in a rotation on the spinor $\hat{\epsilon}=\Omega \epsilon[\underline{4}]$, where $\Omega$ can be found in (2.12). The rotation (3.7) should be accompanied by a mechanism that changes the chirality of the theory. As we will see this will involve the Gamma matrix $\Gamma_{r}$ along the radial direction of the T-dual background.

We next reintroduce the RR fluxes by first describing the Killing spinor equations satisfied by the original type-IIB background prior to the non-Abelian T-duality. Within the confines of our ansatz (2.4), the dilatino variation is 8

$$
\delta \lambda=\frac{1}{2} \not \Phi \epsilon-\frac{1}{24} H \sigma_{3} \epsilon+\frac{1}{2} e^{\Phi}\left[G_{1}\left(i \sigma^{2}\right)+\frac{1}{2}\left(\frac{1}{6} G_{3}-m e^{-3 A} \Gamma^{123}\right) \sigma^{1}\right] \epsilon,
$$

while the gravitino variations along $M_{7}$ and $S^{3}$, respectively, become

$$
\begin{aligned}
\delta \psi_{\mu}= & D_{\mu} \epsilon-\frac{1}{8} H_{\mu \nu \rho} \Gamma^{v \rho} \sigma^{3} \epsilon-\frac{e^{\Phi}}{8}\left[G_{1}\left(i \sigma^{2}\right)+\left(\frac{1}{6} G_{3}-m e^{-3 A} \Gamma^{123}\right) \sigma^{1}\right. \\
& \left.+\frac{e^{-3 A}}{2} G_{2} \Gamma^{123}\left(i \sigma^{2}\right)\right] \Gamma_{\mu} \epsilon
\end{aligned}
$$

and

$$
\begin{aligned}
\delta \psi_{i}= & e^{-A} D_{i}^{S^{3}} \epsilon-\frac{1}{2} \not \partial \Gamma_{i} \epsilon-\frac{e^{\Phi}}{8}\left[G_{1}\left(i \sigma^{2}\right)+\left(\frac{1}{6} G_{3}-m e^{-3 A} \Gamma^{123}\right) \sigma^{1}\right. \\
& \left.+\frac{e^{-3 A}}{2} G_{2} \Gamma^{123}\left(i \sigma^{2}\right)\right] \Gamma_{i} \epsilon .
\end{aligned}
$$

The indices $i=7,8,9$ denote the tangent space directions of $S^{3}$ and $\mu=0, \ldots, 6$ are $M_{7}$ indices. We have used that $D_{i} \epsilon=D_{i}^{S^{3}} \epsilon-\frac{1}{2} e^{A} \not \partial A \Gamma_{i} \epsilon$, so that the covariant derivative $D_{i}^{S^{3}}$ is defined entirely on the three-sphere as the notation indicates.

We would like to rewrite the Killing spinor equations of massive IIA in terms of those

\footnotetext{
${ }^{8}$ All indices in the supersynmmetry variations below as well as in the Gamma matrices are, unless otherwise stated, tangent space indices.
} 
of type-IIB we just described. The key observation is the redefinition of the spinor (3.7). Plugging this into the gravitino variation in the $r$-direction and pulling through the exponential factor $e^{X}$, one obtains

$$
\begin{aligned}
\delta \psi_{r}= & e^{X}\left[\frac{1}{2} \not \partial \Gamma_{r}-\frac{e^{-A}}{4} \Gamma^{\theta \phi} \sigma^{3}+\frac{e^{\Phi}}{8}\left(m e^{-3 A} \Gamma^{\theta \phi} \Gamma_{r}\left(i \sigma^{2}\right)\right.\right. \\
& \left.\left.-G_{1}\left(i \sigma^{2}\right)-\frac{e^{-3 A}}{2} G_{2} \Gamma^{\theta \phi} \Gamma_{r} \sigma^{1}-\frac{1}{6} G_{3} \sigma^{1}\right)\right] \tilde{\epsilon},
\end{aligned}
$$

which is an algebraic condition that will lead to suitable projection conditions on the Killing spinor. One can check that the two known solutions presented in [4] lead to such projections. For the T-dual of $A d S_{5} \times S^{5}$ one finds a single projection condition, while for the T-dual of $A d S_{3} \times S^{3} \times T^{4}$, the vanishing of this gravitino variation is equivalent to the imposition of two projection conditions.

One can then use (3.11) in the dilatino variation of massive IIA which then becomes

$$
\begin{aligned}
\delta \lambda=e^{X} & {\left[\frac{1}{2} \not \partial \Phi-\frac{1}{24} \not H \sigma^{3}\right] \tilde{\epsilon}+\left[\frac{r^{2}+3 e^{4 A}}{r^{2}+e^{4 A}} \Gamma^{r}-\frac{2 r e^{2 A}}{r^{2}+e^{4 A}} \Gamma^{r \theta \phi} \sigma^{3}\right] \delta \psi_{r} } \\
& +e^{X} \frac{e^{\Phi}}{2}\left[-G_{1} \Gamma^{r}\left(i \sigma^{2}\right)+\frac{1}{2}\left(m e^{-3 A} \Gamma^{\theta \phi}\left(i \sigma^{2}\right)-\frac{1}{6} G_{3} \Gamma^{r} \sigma^{1}\right)\right] \tilde{\epsilon} .
\end{aligned}
$$

The gravitino variation along $M_{7}$ may be similarly expressed as

$$
\begin{aligned}
\delta \psi_{\mu}= & e^{X}\left[D_{\mu}-\frac{1}{8} H_{\mu \nu \rho} \Gamma^{v \rho} \sigma^{3}+\frac{e^{\Phi}}{8}\left(e^{-3 A} m \Gamma^{\theta \phi}\left(i \sigma^{2}\right)\right.\right. \\
& \left.\left.-G_{1} \Gamma^{r}\left(i \sigma^{2}\right)-\frac{e^{-3 A}}{2 !} G_{2} \Gamma^{\theta \phi} \sigma^{1}-\frac{1}{3 !} G_{3} \Gamma^{r} \sigma^{1}\right) \Gamma_{\mu}\right] \tilde{\epsilon} .
\end{aligned}
$$

Hence when $\delta \psi_{r}=0$, as required by supersymmetry, then the dilatino variation and the gravitino variations along $M_{7}$ resemble those of type-IIB. It is indeed easy to confirm that the Killing spinor equations on $M_{7}$ are the same and that the Killing spinors are simply rotated as described in [29]. After incorporating the rotations involving $\epsilon^{X}$, one can simply redefine

$$
\tilde{\epsilon}_{+}=\Gamma^{r} \epsilon_{+}, \quad \tilde{\epsilon}_{-}=-\epsilon_{-}, \quad \Gamma^{r \theta \phi}=-\Gamma^{123},
$$

so that, when $\delta \psi_{r}=0$, one maps (3.12) to (3.8) and (3.13) to (3.9). The redefinition in $\epsilon_{+}$means that it flips chirality, in accordance with the Killing spinors for type-IIB. 
The gravitino variation in the direction $\theta$ can then be written as

$$
\begin{aligned}
\delta \psi_{\theta} & =\frac{e^{2 X}}{\sqrt{r^{2}+e^{4 A}}}\left(e^{2 A} \Gamma^{r \phi} \sigma^{3}-\frac{e^{4 A}}{r} \Gamma^{r \theta}\right) \delta \psi_{r} \\
& +\frac{e^{X} \sqrt{r^{2}+e^{4 A}}}{r}\left[e^{-A} \partial_{\theta}+\frac{1}{2} \Gamma^{\theta \mu} \partial_{\mu} A+\frac{e^{-A}}{4} \Gamma^{r \phi} \sigma^{3}+\frac{e^{\Phi}}{8}\left(-m e^{-3 A} \Gamma^{\phi}\left(i \sigma^{2}\right)\right.\right. \\
& \left.\left.-G_{1} \Gamma^{r \theta}\left(i \sigma^{2}\right)+\frac{e^{-3 A}}{2} G_{2} \Gamma^{\phi} \sigma^{1}-\frac{1}{6} G_{3} \Gamma^{r \theta} \sigma^{1}\right)\right] \tilde{\epsilon},
\end{aligned}
$$

where of course the index in $\partial_{\theta}$ is curved, or simplifying further, as

$$
\delta \psi_{\theta}=e^{4 X} \Gamma^{r \theta} \delta \psi_{r}+\frac{e^{-A} \sqrt{r^{2}+e^{4 A}}}{r}\left(\partial_{\theta}+\frac{1}{2} \Gamma^{r \phi} \sigma^{3}\right) \tilde{\epsilon} .
$$

There is a similar variation for the remaining direction $\phi$ along $S^{2}$, so that we may write compactly that

$$
\delta \psi_{\alpha}=e^{2 X} \Gamma_{r} \Gamma_{\alpha} \delta \psi_{r}+\frac{e^{-A} \sqrt{r^{2}+e^{4 A}}}{r}\left(D_{\alpha}+\frac{1}{2} \epsilon_{\alpha \beta} \Gamma_{r} \Gamma_{\beta} \sigma_{3}\right) \tilde{\epsilon},
$$

where $\alpha, \beta$ are tangent space indices along the unit 2-sphere directions $\theta$ and $\phi$ of the Tdual background and $\epsilon_{\alpha \beta}$ is the two-dimensional antisymmetric tensor with $\epsilon_{\theta \phi}=1$. According to our normalizations $R_{\alpha \beta}=g_{a \beta}$. Hence, one can readily check that the integrability condition of the $\delta \psi_{\alpha}=0$ equation, arising from $\left[D_{\mu}, D_{\nu}\right] \epsilon=\frac{1}{4} R_{\mu v a b} \Gamma^{a b} \epsilon$, is indeed satisfied without a requirement for any extra projection condition.

Hence upon satisfying $\delta \psi_{r}=0$ in (3.11) we may map the massive IIA Killing spinor equations to those of type-IIB. In fact, this equation becomes that of type-IIB along $S^{3}$ in (3.10) provided that in this theory the Killing spinors satisfy

$$
D_{i}^{S^{3}} \epsilon=\frac{1}{4} \Gamma_{123} \Gamma_{i} \epsilon,
$$

which is the Killing spinor equation on $S^{3}$. This is not a trivial statement in the sense that there should be extra projections imposed on it in order to be fulfilled. The solu- 
tion to (3.18) in the coordinate system (3.2) is readily found to be9

$$
\epsilon=\exp \left(\frac{\theta}{2} \Gamma_{\phi \psi}\right) \exp \left(-\frac{\phi+\psi}{2} \Gamma_{\theta \psi}\right) \epsilon_{0}
$$

where $\epsilon_{0}$ is a spinor that could depend on the $M_{7}$ coordinates. Hence, given a solution of the type-IIB Killing spinor equations we should impose suitable projections so that the solution eventually assumes the form (3.20). For example, for the case of the nonAbelian T-dual to flat spacetime we easily see that by imposing the projection

$$
\Gamma_{x} \epsilon_{0}=-\Gamma_{\theta \phi \psi} \epsilon_{0}
$$

into (3.3) we indeed obtain (3.20). In fact we may proceed further and show that (3.20) implies that the Lorentz-Lie (equivalently the Kosmann) derivative on the Killing spinor of the original type-IIB theory vanishes. We recall that the latter defines the action of a vector on a spinor as [44, 45, 46]

$$
\mathcal{L}_{k} \epsilon=k^{\mu} D_{\mu} \epsilon+\frac{1}{4} D_{\mu} k_{\nu} \Gamma^{\mu \nu} \epsilon
$$

This derivation maps spinors to spinors and if $k^{\mu} \partial_{\mu}$ is a Killing vector then they obey the Lie-algebra of the associated symmetry group. In our case this symmetry algebra is generated by the left and right invariant vector fields corresponding to the MaurerCartan forms with structure constants $\epsilon_{a b c}$ and $-\epsilon_{a b c}$, respectively. Recall also that a Killing vector remains so in all conformally related metrics. Since the original Killing spinor corresponds to a background with $S U(2)_{L} \times S U(2)_{R}$ symmetry it transforms in the direct product of the spinor representations of the two factors. The non-Abelian T-dual background preserves just the $S U(2)_{R}$ factor. This is encoded in the vanishing of the Kosmann derivative (3.22) for the right invariant Killing vectors. After some algebraic manipulations one shows that demanding the latter condition implies (3.18), thus fully justifying the use of the Kosmann derivative in the present context as introduced in [4].

Hence, our general conclusion is that, within our class of type-IIB backgrounds (2.1), (2.4) with $S O(4)$ symmetry, a non-Abelian T-duality transformation with respect to the

\footnotetext{
${ }^{9}$ We use the frame

$$
e^{\theta}=2 d \theta, \quad e^{\phi}=2 \cos \theta d \phi, \quad e^{\psi}=-2 \sin \theta d \psi,
$$
}

where the introduction of the minus sign is in accordance with (3.14). 
$S U(2)$ subgroup giving rise to the massive IIA background (2.2) and (2.16), reduces supersymmetry by half if the Killing spinor transforms under the $S U(2)$ factor that we use to perform the T-duality transformation, or leaves it intact if it does not transform at all.

\section{Massive IIA non-Abelian T-duals}

For completeness we will also consider $S O(4)$ symmetric backgrounds in massive IIA supergravity and their non-Abelian duals with respect to an $S U(2)$ subgroup. We begin by establishing an $S O(4)$ invariant spacetime ansatz where we simply retain the singlets. For the NS sector fields the metric is still given by (2.1) as in the type-IIB case, we omit the presence of a $B$-field along $S^{3}$ and the dilaton may depend only on coordinates of $M_{7}$. The massive IIA ansatz for the fluxes is

$$
\begin{aligned}
& F_{0}=m, \\
& F_{2}=G_{2}, \\
& F_{4}=G_{4}+G_{1} \wedge \operatorname{Vol}\left(S^{3}\right) .
\end{aligned}
$$

As for type-IIB one can consistently reduce the equations of motion of massive IIA on $S^{3}$ and obtain eventually in the Einstein frame (B.21). Using the redefinition (2.6) one finds the following seven-dimensional action

$$
\begin{aligned}
\mathcal{L}_{\text {Einstein }} & =R-20(\partial \Phi)^{2}-3(\partial A)^{2}-\frac{1}{12} e^{-8 \Phi} H^{2} \\
- & \frac{1}{2}\left(m^{2} e^{14 \Phi+3 A}-3 e^{4 \Phi-2 A}+e^{10 \Phi-3 A} G_{1}^{2}+\frac{1}{2} e^{6 \Phi+3 A} G_{2}^{2}+\frac{1}{4 !} e^{3 A-2 \Phi} G_{4}^{2}\right) \\
- & G_{4} \wedge G_{1} \wedge B+\frac{m}{3} G_{1} \wedge B^{3}+\frac{1}{2} B^{2} \wedge d C_{1} \wedge G_{1}
\end{aligned}
$$

where

$$
G_{1}=d C_{0}, \quad G_{2}=d C_{1}+m B, \quad G_{4}=d C_{3}-H \wedge C_{1}+\frac{m}{2} B \wedge B .
$$

The RR fluxes transform according to (2.11) with the expressions for $P$ and $\hat{P}$ interchanged since now the original background is in massive IIA and the final in type-IIB supergravity. Omitting the details, which are given in Appendix B.2, we find that 


$$
\begin{aligned}
& \hat{F}_{1}=-G_{1}-m r d r \\
& \hat{F}_{3}=e^{3 A} \star_{7} G_{4}-r d r \wedge G_{2}-\frac{r^{3}}{r^{2}+e^{4 A}} G_{1} \wedge \operatorname{Vol}\left(S^{2}\right)+\frac{m r^{2} e^{4 A}}{r^{2}+e^{4 A}} d r \wedge \operatorname{Vol}\left(S^{2}\right), \\
& \hat{F}_{5}=\frac{r^{2} e^{3 A}}{r^{2}+e^{4 A}}\left(r \star_{7} G_{4}+e^{A} d r \wedge G_{2}\right) \wedge \operatorname{Vol}\left(S^{2}\right)-e^{3 A} \star_{7} G_{2}-r d r \wedge G_{4} .
\end{aligned}
$$

Finally, we note that an analysis along the lines of section 3 leads to the same conclusion that supersymmetry is broken by half in this case as well.

\section{Explicit examples}

In this paper we have placed non-Abelian T-duality on a firmer footing. Instead of being confined to near-horizon solutions [4, 5], we are now in a position to generate large families of solutions on the proviso that the original solution has an $S^{3}$ factor. We can now simply match the original solution to our ansatz and read off the non-Abelian T-dual. We discuss below three such examples.

\subsection{PP-wave}

We begin by warming up with the maximally supersymmetric pp-wave in type-IIB supergravity [30]. Here we have $S O(4) \times S O(4)$ isometry, so we have a choice of two three-spheres to T-dualise. Isolating the two $S^{3}$ 's, the solution may be written as

$$
\begin{aligned}
d s^{2} & =2 d x^{+} d x^{-}-\mu^{2}\left[y^{2}+z^{2}\right]\left(d x^{+}\right)^{2}+d y^{2}+\frac{1}{4} y^{2} d s^{2}\left(S^{3}\right)+d z^{2}+\frac{1}{4} z^{2} d s^{2}\left(\tilde{S}^{3}\right) \\
F_{5} & =\frac{1}{2} \mu y^{3} d x^{+} \wedge d y \wedge \operatorname{Vol}\left(S^{3}\right)+\frac{1}{2} \mu z^{3} d x^{+} \wedge d z \wedge \operatorname{Vol}\left(\tilde{S}^{3}\right)
\end{aligned}
$$

where again we use for the three-spheres the normalization $R_{i j}=\frac{1}{2} g_{i j}$. We can now read off the field content in seven dimensions

$$
G_{2}=\frac{1}{2} \mu y^{3} d x^{+} \wedge d y, \quad e^{A}=\frac{y}{2} .
$$


We can then generate the T-dual solution of type-IIA

$$
\begin{aligned}
d s^{2} & =2 d x^{+} d x^{-}-\mu^{2}\left[y^{2}+z^{2}\right]\left(d x^{+}\right)^{2}+d y^{2}+\frac{4}{y^{2}} d r^{2}+\frac{4 r^{2} y^{2}}{16 r^{2}+y^{4}} d s^{2}\left(S^{2}\right) \\
& +d z^{2}+z^{2} d s^{2}\left(\tilde{S}^{3}\right), \\
\Phi & =-\frac{1}{2} \ln \left[\frac{y^{2}}{4}\left(r^{2}+\frac{y^{4}}{16}\right)\right], \quad B=\frac{16 r^{3}}{16 r^{2}+y^{4}} \operatorname{Vol}\left(S^{2}\right), \\
F_{2} & =-\frac{1}{2} \mu y^{3} d x^{+} \wedge d y, \quad F_{4}=-\frac{8 r^{3} \mu y^{3}}{16 r^{2}+y^{4}} d x^{+} \wedge d y \wedge \operatorname{Vol}\left(S^{2}\right) .
\end{aligned}
$$

It is also easy to see that supersymmetry is broken by one half. Typically pp-waves of this form always preserve 16 supersymmetries in the kernel of $\Gamma^{+}$. Plugging the solution into (3.11) one notes that these Killing spinors are subject to a projection condition $\Gamma^{y r} \theta \phi \sigma^{3} \tilde{\epsilon}_{+}=\tilde{\epsilon}_{+}$, where we have used the subscript to denote Killing spinors satisfying $\Gamma^{+} \tilde{\epsilon}_{+}=0$. The other sixteen Killing spinors not killed by $\Gamma^{+}$will also be subject to the same projector, so both standard Killing spinors and supernumerary Killing spinors are cut by one half. Indeed, this should not come as a surprise. In the process of constructing the non-Abelian T-dual we have deformed the original solution so that the Ricci tensor has non-zero components other than $R_{++}$.

We can now do the reverse transformation by reading off the transformation from section 4. There is no need to rescale the fluxes as the fluxes are now along $M_{7}$ only. The warp factor is then $e^{A}=\frac{z}{2}$ and the T-dual geometry in type-IIB, after doing two non-Abelian T-dualities is

$$
\begin{aligned}
d s^{2} & =2 d x^{+} d x^{-}-\mu^{2}\left[y^{2}+z^{2}\right]\left(d x^{+}\right)^{2}+d y^{2}+\frac{4}{y^{2}} d r^{2}+\frac{4 r^{2} y^{2}}{16 r^{2}+y^{4}} d s^{2}\left(S^{2}\right) \\
& +d z^{2}+\frac{4}{z^{2}} d \tilde{r}^{2}+\frac{4 \tilde{r}^{2} z^{2}}{16 \tilde{r}^{2}+z^{4}} d s^{2}\left(\tilde{S}^{2}\right), \\
e^{-2 \Phi} & =\frac{(y z)^{2}}{16}\left(r^{2}+\frac{y^{4}}{16}\right)\left(\tilde{r}^{2}+\frac{z^{4}}{16}\right), \\
B & =\frac{16 r^{3}}{16 r^{2}+y^{4}} \operatorname{Vol}\left(S^{2}\right)+\frac{16 \tilde{r}^{3}}{16 \tilde{r}^{2}+z^{4}} \operatorname{Vol}\left(\tilde{S}^{2}\right), \\
F_{3} & =\frac{z^{3}}{8} \star_{7} F_{4}-\tilde{r} F_{2} \wedge d \tilde{r}, \\
F_{5} & =(1+*)\left[\frac{2 z^{3} \tilde{r}^{3}}{16 \tilde{r}^{2}+z^{4}} \star_{7} F_{4}+\frac{z^{4} \tilde{r}^{2}}{16 \tilde{r}^{2}+z^{4}} F_{2} \wedge d \tilde{r}\right] \wedge \operatorname{Vol}\left(\tilde{S}^{2}\right),
\end{aligned}
$$


where $F_{2}$ and $F_{4}$ are given above in (5.3). One can check that the fluxes are of the appropriate form so that we still have symmetry under the exchange $(r, y) \leftrightarrow(\tilde{r}, z)$.

\subsection{Type-IIB backgrounds with $S O(4) \times S O(4) \times \mathbb{R}$ isometry}

In this section we consider the class of backgrounds constructed in [31] that correspond to $\frac{1}{2}$-BPS states. These contain two round three-spheres and a time-like Killing vector. The metric is given by

$$
d s^{2}=-h^{-2}\left(d t+V_{i} d x^{i}\right)^{2}+h^{2}\left(d y^{2}+d x^{i} d x^{i}\right)+\frac{1}{4} y e^{G} d s^{2}\left(S^{3}\right)+\frac{1}{4} y e^{-G} d s^{2}\left(\tilde{S}^{3}\right),
$$

where $i=1,2$ and $h, V_{i}$ and $G$ are functions of the $x^{i}$ s spanning an $\mathbb{R}^{2}$ and $y \geqslant 0$. They are related through the Killing spinor equations by

$$
\begin{aligned}
& h^{-2}=2 y \cosh G, \quad y \partial_{y} V_{i}=\epsilon_{i j} \partial_{j} z, \\
& y\left(\partial_{i} V_{j}-\partial_{j} V_{i}\right)=\epsilon_{i j} \partial_{y} z, \quad z=\frac{1}{2} \tanh G .
\end{aligned}
$$

The non-trivial 5-form field strength is

$$
F_{5}=G_{2} \wedge \operatorname{Vol}\left(S^{3}\right)+\tilde{G}_{2} \wedge \operatorname{Vol}\left(\tilde{S}^{3}\right),
$$

where the two-forms $G_{2}$ and $\tilde{G}_{2}$ are along $t, x_{i}$ and $y$ and are given by

$$
\begin{aligned}
& 8 G_{2}=d B_{t} \wedge(d t+V)+B_{t} d V+d \hat{B}, \\
& 8 \tilde{G}_{2}=d \tilde{B}_{t} \wedge(d t+V)+\tilde{B}_{t} d V+d \hat{\tilde{B}},
\end{aligned}
$$

with

$$
\begin{aligned}
& B_{t}=-\frac{1}{4} y^{2} e^{2 G}, \quad \tilde{B}_{t}=-\frac{1}{4} y^{2} e^{-2 G}, \\
& d \hat{B}=-\frac{1}{4} y^{3} \star_{3} d\left(\frac{z+1 / 2}{y^{2}}\right), \quad d \hat{B}=-\frac{1}{4} y^{3} \star_{3} d\left(\frac{z-1 / 2}{y^{2}}\right),
\end{aligned}
$$

where the Hodge star is with respect to the three-dimensional metric with coordinates the $x^{i}$ s and $y$. The whole background can then be determined in terms of the function $z\left(x^{1}, x^{2}, y\right)$ satisfying

$$
\partial_{i} \partial_{i} z+y \partial_{y}\left(\frac{\partial_{y} z}{y}\right)=0
$$


and arising as the integrability condition for the differential equations in (5.6). Solutions to this are easily found by realizing that $\Phi=\frac{z}{y^{2}}$ satisfies the six-dimensional Laplace equation with $S O(4)$ rotational symmetry and $y$ being the radial coordinate.

An important issue in this class of backgrounds is regularity [31]. In order to avoid the singularity at $y=0$, the function $z\left(x^{1}, x^{2}, 0\right)$ must only take the two possible values $\pm \frac{1}{2}$ which are related to the symmetry of the background under the exchange of the two round 3-spheres. The general solution of (5.10) must then satisfy these boundary conditions in order to be regular. Near $z=\frac{1}{2}$, we have that $z=\frac{1}{2}-e^{-2 G}$ for $G$ large. Simultaneously, as $y \rightarrow 0$, the function $h^{-2} \simeq y e^{G}$ remains finite. Then the part of the metric spanned by $y$ and the two 3-spheres behaves in this limit as

$$
\frac{1}{4} h^{-2} d s^{2}\left(S^{3}\right)+h^{2}\left(d y^{2}+\frac{y^{2}}{4} d s^{2}\left(\tilde{S}^{3}\right)\right) .
$$

One can also show that $V$ remains finite. For $z=-\frac{1}{2}$ the same holds with the two three-spheres interchanged. The $\mathbb{R}^{2}$ plane has a natural interpretation as the phase space of one-dimensional fermions in a harmonic potential [31]. It is filled by quantum Hall droplets where the fermions are localized. Their density $\rho\left(x^{1}, x^{2}\right)=$ $\frac{1}{2}-z\left(x^{1}, x^{2}, 0\right)$ is a step function, i.e. it takes the value 1 inside the droplets and 0 outside.

The background (5.5), (5.7) is of the general type (2.1), (2.4) with

$$
e^{A}=\frac{1}{2} \sqrt{y} e^{G / 2}, \quad m=0, \quad G_{1}=0, \quad G_{3}=0
$$

and non-vanishing $G_{2}$. The metric of the non-Abelian T-dual solution is

$$
\begin{aligned}
d s^{2}= & -h^{-2}\left(d t+V_{i} d x^{i}\right)^{2}+h^{2}\left(d y^{2}+d x^{i} d x^{i}\right) \\
& +4 y^{-1} e^{-G} d r^{2}+\frac{4 r^{2} y e^{G}}{16 r^{2}+y^{2} e^{2 G}} d s^{2}\left(S^{2}\right)+\frac{1}{4} y e^{-G} d s^{2}\left(\tilde{S}^{3}\right),
\end{aligned}
$$

supported by

$$
\begin{aligned}
& \Phi=-\frac{1}{2} \ln \left(y e^{G}\left(16 r^{2}+y^{2} e^{2 G}\right)\right), \quad B=\frac{16 r^{3}}{16 r^{2}+y^{2} e^{2 G}} \operatorname{Vol}\left(S^{2}\right), \\
& F_{2}=-G_{2}, \quad F_{4}=-\frac{16 r^{3}}{16 r^{2}+y^{2} e^{2 G}} G_{2} \wedge \operatorname{Vol}\left(S^{2}\right) .
\end{aligned}
$$

This background is singular at $y=0$, where the radii of both the two-and the three- 
spheres vanish, unless $z=\frac{1}{2}$. In this limit the part of the metric (5.13) spanned by $y, r$ and the 2- and 3-spheres behaves as

$$
h^{2}\left[d y^{2}+4 d r^{2}+\frac{4 r^{2}}{1+16 h^{4} r^{2}} d s^{2}\left(S^{2}\right)+\frac{y^{2}}{4} d s^{2}\left(\tilde{S}^{3}\right)\right],
$$

which is non-singular. The same happens for the rest of NS-NS and RR fields. On the other hand if $z=-\frac{1}{2}, h^{-2} \simeq y e^{-G}$ and the metric and dilaton fields are singular. Therefore the dual solution is non-singular only outside the droplets (in the original description) where $z=\frac{1}{2}$. This was expected since after the dualization the symmetry under the exchange of the two 3-spheres is lost. In a similar fashion, if we perform the non-Abelian T-duality transformation on the $\tilde{S}^{3}$, we get a background that is singular

outside the droplets where $z=\frac{1}{2}$ and non-singular inside them where $z=-\frac{1}{2}$. Obviously, if we T-dualize with respect to both round three-spheres we obtain a singular background everywhere in $\mathbb{R}^{2}$. These general results are in agreement with the conclusions of [4], where the non-Abelian T-dual of $A d S_{5} \times S^{5}$ with respect to the $S U(2)$ subgroup of the $S O(6)$ isometry group of the five-sphere was constructed. Also we point out that there are interesting cases where the above regularity conditions are violated. Notably, if we use in place of the step function mentioned above, fermion distributions at finite temperature [47] or in describing in the present context the socalled superstar solutions as in [48].

Finally, given that the $S O(4) \times S O(4)$ symmetric massive deformation of ABJM considered in [31, 49] belongs as well to the general class of backgrounds, in this case of type-IIA, considered in this paper, we can use non-Abelian T-duality to generate a type-IIB solution with non-vanishing $F_{i}$ for $i=1,3,5$, a NS $B$-field and a metric with the same $y, r, 2$-sphere and 3 -sphere components as in (5.13). We omit the details given the similarity with the present solution.

\subsection{Lifshitz symmetry solutions}

A particularly interesting class of geometries involves those exhibiting Lifshitz symmetry [50]. For concreteness, we will consider the solutions of [32] based on an $S^{5}$ internal geometry, but will follow the notation of [51].

According to [51], Lifshitz solutions with dynamical exponent $z=2$ in Einstein frame 
may be written as

$$
\begin{aligned}
d s^{2} & =r^{2}\left[2 d x^{+} d x^{-}+d x_{1}^{2}+d x_{2}^{2}\right]+\frac{d r^{2}}{r^{2}}+f\left(d x^{+}\right)^{2}+d s^{2}\left(E_{5}\right) \\
F_{5} & =4(1+*) \operatorname{Vol}\left(E_{5}\right) \\
G & =d x^{+} \wedge W, \quad P=g d x^{+}
\end{aligned}
$$

where $W, f$ and $g$ satisfy

$$
d x^{+} \wedge d W=d *_{E} W=0, \quad-\nabla_{E}^{2} f+4 f=4|g|^{2}+|W|_{E}^{2}
$$

Here $E_{5}$ denotes a compact Einstein manifold and we are using complex notation for the three-form and the axion-dilaton 10 .

The above requirement that $W$ be harmonic, (5.16), means that there are no solutions with non-zero $W$ for $S^{5}$, but supersymmetric solutions [51] do exist for Sasaki-Einstein spaces such as $T^{1,1}$ [52] and $Y^{p, q}$ [53] which are topologically $S^{2} \times S^{3}$. However, in spite of these spaces having the correct topology, neither possess a round $S^{3}$ fitting into our ansatz, so we confine ourselves to $W=0$ with $E_{5}$ being $S^{5}$. To recover the solution of [32] one simply takes $W=0$ with $f$ and $g$ only depending on the coordinate $x^{+}$. A further subclass considers the case where $f$ is a constant, which following [51], we also take to be the identity. In this case from (5.16) we have $g=e^{i \beta}$, where $\beta \in[0, \pi / 2]$. Then introducing the usual fibration of $S^{5}$,

$$
d s^{2}=d \tilde{\theta}^{2}+\sin ^{2} \tilde{\theta} d \tilde{\phi}^{2}+\cos ^{2} \tilde{\theta} d s^{2}\left(S^{3}\right),
$$

we can perform a non-Abelian T-duality to get the type-IIA solution 11 After transforming to the string frame, one can simply read off the T-dual solution from the formulae in section 2, giving the following expressions

$$
\begin{aligned}
e^{\Phi} & =e^{2 \cos \beta x^{+}}, \\
e^{A} & =\frac{1}{2} e^{\frac{1}{2} \cos \beta x^{+}} \cos \tilde{\theta}, \\
G_{1} & =2 \sin \beta e^{-2 \cos \beta x^{+}} d x^{+},
\end{aligned}
$$

\footnotetext{
${ }^{10}$ In terms of more usual string theory variables, these may be written as $G=i e^{\frac{\Phi}{2}}\left(\tau d B-d C_{2}\right)$, $P=\frac{i}{2} e^{\Phi} d C_{0}+\frac{1}{2} d \Phi$, where $\tau=C_{0}+i e^{-\Phi}$.

${ }^{11}$ Note that in this case the $S^{3}$ metric is normalized such that $R_{i j}=2 g_{i j}$.
} 


$$
G_{2}=\frac{1}{2} \cos ^{3} \tilde{\theta} \sin \tilde{\theta} d \tilde{\theta} \wedge d \tilde{\phi},
$$

with $G_{3}=m=0$ and where we have introduced tildes to differentiate angles on $M_{7}$ from angles on $S^{3}$.

One final interesting point pertains to supersymmetry. As is discussed in [51], these solutions prior to T-duality generically preserve two supersymmetries which are further enhanced to eight supersymmetries when $E_{5}=S^{5}$ [54]. These eight supersymmetries are those preserved by $\Gamma^{+} \epsilon=0, \Gamma^{+-12} \epsilon=i \epsilon$, where we have used complex spinors $\epsilon=\epsilon_{1}+i \epsilon_{2}$. The non-Abelian solution is subject to the additional projection found by imposing (3.11) and therefore it preserves four supercharges.

\section{Concluding remarks}

In this paper we have put non-Abelian duality on a firmer footing by showing that it relates backgrounds that give rise to the same consistent truncation in seven dimensions. An important drawback of the original $\sigma$-model derivation in [3] was the fact that it was not possible to reconstruct the original background from the dual one due to its lack of isometries. This problem is sorted out in the supergravity formulation, where any solution of the seven-dimensional theory can be uplifted to both type-IIB and massive IIA supergravities. As we have seen, provided the original type-IIB solution has an RR $F_{3}$-flux along $S^{3}$, a solution to massive IIA results. This provides quite a general set-up in which to generate solutions to massive type-IIA supergravity.

We have also made a step in understanding supersymmetry breaking under nonAbelian T-duality. Through a mapping of the Killing spinor equations and an expected redefinition of the Killing spinors, we have seen that the supersymmetry conditions get mapped modulo an additional consistent condition that can break half the supersymmetry.

Since a $B$-field along the $S U(2)$ directions of the dual background is also generated, non-Abelian duality can be useful as well as a way to produce solutions with a nonvanishing $B$. This raises an open question about D-brane probe dynamics [55] and the role of the $B$-field in overcoming natural repulsion between probe branes (see [56]). Related to D-branes and their T-duals is a very pertinent question regarding the charges of the T-dual geometry. Since the resulting geometry is non-compact, some 
form of regularization of the flux integrals will be required. On top of this, we can ask if there is an AdS/CFT picture and if the large N limit of the solutions could be useful in the spirit of [57] to describe non-commutative gauge theories. Furthermore, if a dual CFT picture can be understood, since supersymmetry is broken by a half, it would certainly be interesting to understand this from the CFT point of view.

As we have already mentioned, we expect that more general non-Abelian duality transformations based on larger symmetry groups or acting with fixed points will also fit into this picture. The most straightforward extension is the construction of non-Abelian SU(2) duals in which the $S U(2)$ acts with fixed points. In this case, and based on the examples presented in [5], we conjecture that the original and dual backgrounds will also give rise to consistent truncations to lower-dimensional theories. On that, we note that as our reduction on $S^{3}$ from type-IIB does not give rise to the expected maximal supergravity in seven dimensions [7], it would be satisfactory if the full reduction could be identified, or if the origin of the massive multiplets could be elucidated.

We have seen in subsection 2.3 that in order to reproduce (2.7) from the action of massive IIA on the T-dual background with $\mathbb{R} \times S^{2}$ topology, we have to take a correlated limit in which the coupling constant of the ten-dimensional theory is taken infinitely large. As already mentioned in the main text, in analogy with the exact CFT models investigated in [43] this implies that the non-Abelian T-dual background effectively captures states corresponding to group theory representations with infinitely high highest weight. This also resonates with a result of [4] in which the non-Abelian Tdual of an $S U(2) \subset S O(6)$ of the $A d S_{5} \times S^{5}$ background gave rise to a solution whose M-theory lift captures generic features of the geometries proposed in [59] as gravity duals to $N=2$ gauge theories. These features correspond to a zooming of the generic geometries presumably associated to high spin states in the dual CFT. It is important to pursue work that substantiates further this idea.

Finally, it would be interesting to examine the effect of non-Abelian T-duality on supergravity solutions with interesting four-dimensional cosmological interpretations. Since T-duality breaks the $S O(4)$ symmetry of solutions with homogeneity and isotropy down to $S O(3)$, it implies that the homogeneity is lost. The important physical question in the present context is to investigate the Big-Bang scenario and the fate of the initial singularity. 


\section{Acknowledgements}

We are grateful to Bert Janssen, Hai Lin, Hong Lu, Patrick Meessen, Tomás Ortín and Henning Samtleben for useful discussions. The research of G. Itsios has been co-financed by the ESF and Greek national funds through the Operational Program "Education and Lifelong Learning" of the NSRF - Research Funding Program: "Heracleitus II. Investing in knowledge in society through the European Social Fund". He would also like to thank the CFP of University of Porto for hospitality within the framework of the LLP/Erasmus Placements 2011-2012. Y. Lozano and E. Ó Colgáin have been partially supported by the research grants MICINN-09-FPA2009-07122 and MEC-DGI-CSD2007-00042.

\section{A Review of type-II supergravities}

In this appendix we review aspects of type-II supergravities relevant to this work.

\section{A.1 Type-IIB supergravity}

The action of type-IIB supergravity is given by

$$
\begin{aligned}
S_{\mathrm{IIB}}=\frac{1}{2 \kappa^{2}} \int_{M_{10}} \sqrt{-g}\left[e^{-2 \Phi}\left(R+4(\partial \Phi)^{2}-\frac{H^{2}}{12}\right)-\frac{1}{2}\left(F_{1}^{2}+\frac{F_{3}^{2}}{3 !}+\frac{1}{2} \frac{F_{5}^{2}}{5 !}\right)\right] \\
-\frac{1}{2} C_{4} \wedge H \wedge d C_{2}
\end{aligned}
$$

where the field strengths in terms of the potentials are

$$
H=d B, \quad F_{1}=d C_{0}, \quad F_{3}=d C_{2}-C_{0} H, \quad F_{5}=d C_{4}-H \wedge C_{2} .
$$

In addition, $F_{5}$ has to be self-dual. The Bianchi identities are

$$
d H=0, \quad d F_{1}=0, \quad d F_{3}=H \wedge F_{1}, \quad d F_{5}=H \wedge F_{3} .
$$

Einstein's equations that follow from varying the metric are

$$
R_{\mu \nu}+2 D_{\mu} D_{\nu} \Phi-\frac{1}{4} H_{\mu \nu}^{2}
$$




$$
=e^{2 \Phi}\left[\frac{1}{2}\left(F_{1}^{2}\right)_{\mu v}+\frac{1}{4}\left(F_{3}^{2}\right)_{\mu v}+\frac{1}{96}\left(F_{5}\right)_{\mu \nu}^{2}-\frac{1}{4} g_{\mu \nu}\left(F_{1}^{2}+\frac{1}{6} F_{3}^{2}\right)\right] .
$$

Note the fact that $F_{5}^{2}=0$ due to the self-duality condition $\star F_{5}=F_{5}$. The equation coming from varying the dilaton is

$$
R+4 D^{2} \Phi-4(\partial \Phi)^{2}-\frac{1}{12} H^{2}=0 .
$$

Finally, from the variation of the various fluxes we obtain

$$
\begin{aligned}
& d\left(e^{-2 \Phi} \star H\right)-F_{1} \wedge \star F_{3}-F_{3} \wedge F_{5}=0, \\
& d \star F_{1}+H \wedge \star F_{3}=0, \\
& d \star F_{3}+H \wedge F_{5}=0, \\
& d \star F_{5}-H \wedge F_{3}=0 .
\end{aligned}
$$

The equation of motion for $F_{5}$ is equivalent to the Bianchi identity for the 5-form, as it is self-dual.

\section{A.2 Massive IIA supergravity}

Having introduced type-IIB we now turn to massive type-IIA supergravity. In making the transition from type-IIA supergravity to massive IIA, one simply modifies the definitions of the field strengths by introducing a mass parameter $m$ as

$$
H=d B, \quad F_{2}=d C_{1}+m B, \quad F_{4}=d C_{3}-H \wedge C_{1}+\frac{m}{2} B \wedge B .
$$

The relative coefficients are fixed so that the field strengths are invariant under the gauge transformations

$$
\delta B=d \Lambda, \quad \delta C_{1}=-m \Lambda, \quad \delta C_{3}=-m \Lambda \wedge B,
$$

where $\Lambda$ is a one-form. The Bianchi identities become

$$
d H=0, \quad d F_{2}=m H, \quad d F_{4}=H \wedge F_{2},
$$

which means that $m$ is like an expectation value for an $F_{0}$ term. The action is the same as in type-IIA theory, but with the new definitions for the field strengths. The 
topological term can therefore be written as

$$
-\frac{1}{2} \int_{M_{11}} F_{4} \wedge F_{4} \wedge H=\cdots=-\frac{1}{2} \int_{M_{10}} d C_{3} \wedge d C_{3} \wedge B+\frac{m}{3} d C_{3} \wedge B^{3}+\frac{m^{2}}{20} B^{5},
$$

using an obvious notation for the powers of the forms.

Hence, the action of the massive IIA supergravity is

$$
\begin{aligned}
S_{\text {Massive IIA }}= & \frac{1}{2 \kappa^{2}} \int_{M_{10}} \sqrt{-g}\left[e^{-2 \Phi}\left(R+4(\partial \Phi)^{2}-\frac{H^{2}}{12}\right)-\frac{1}{2}\left(m^{2}+\frac{F_{2}^{2}}{2}+\frac{F_{4}^{2}}{4 !}\right)\right] \\
& -\frac{1}{2}\left(d C_{3} \wedge d C_{3} \wedge B+\frac{m}{3} d C_{3} \wedge B^{3}+\frac{m^{2}}{20} B^{5}\right)
\end{aligned}
$$

Einstein's equations are

$$
\begin{aligned}
R_{\mu \nu}+2 D_{\mu} D_{\nu} \Phi & -\frac{1}{4} H_{\mu \nu}^{2} \\
& =e^{2 \Phi}\left[\frac{1}{2}\left(F_{2}^{2}\right)_{\mu \nu}+\frac{1}{12}\left(F_{4}^{2}\right)_{\mu \nu}-\frac{1}{4} g_{\mu \nu}\left(\frac{1}{2} F_{2}^{2}+\frac{1}{24} F_{4}^{2}+m^{2}\right)\right] .
\end{aligned}
$$

The flux equations are

$$
\begin{aligned}
& d\left(e^{-2 \Phi} \star H\right)-F_{2} \wedge \star F_{4}-\frac{1}{2} F_{4} \wedge F_{4}=m \star F_{2}, \\
& d \star F_{2}+H \wedge \star F_{4}=0, \\
& d \star F_{4}+H \wedge F_{4}=0 .
\end{aligned}
$$

This set of equations is consistent with the Bianchi identities as it can be seen by applying to each one of them the exterior derivative. The dilaton equation (A.5) is the same as before.

\section{A.3 Supersymmetry}

Our conventions for supersymmetry variations follow those of [29]. To package these variations we find it handy to introduce a Killing spinor comprising of real MajoranaWeyl spinors

$$
\epsilon=\left(\begin{array}{c}
\epsilon_{+} \\
\epsilon_{-}
\end{array}\right) .
$$


In type-IIB we have $\Gamma^{11} \epsilon=\epsilon$, while in type-IIA the conventions are such that $\Gamma^{11} \epsilon_{ \pm}=$ $\mp \epsilon_{ \pm}$. Using Pauli matrices, the type-IIA Killing spinor equations can be written as

$$
\begin{aligned}
& \delta \lambda=\frac{1}{2} \not \supset \Phi \epsilon-\frac{1}{24} \not H \sigma_{3} \epsilon+\frac{1}{8} e^{\Phi}\left[5 m \sigma^{1}+\frac{3}{2} \not_{2}\left(i \sigma^{2}\right)+\frac{1}{24} \not F_{4} \sigma^{1}\right] \epsilon, \\
& \delta \psi_{\mu}=D_{\mu} \epsilon-\frac{1}{8} H_{\mu \nu \rho} \Gamma^{\nu \rho} \sigma^{3} \epsilon+\frac{e^{\Phi}}{8}\left[m \sigma^{1}+\frac{1}{2} \not F_{2}\left(i \sigma^{2}\right)+\frac{1}{24} \not F_{4} \sigma^{1}\right] \Gamma_{\mu} \epsilon,
\end{aligned}
$$

where $D_{\mu} \epsilon=\partial_{\mu} \epsilon+\frac{1}{4} \omega_{\mu}^{a b} \Gamma_{a b} \epsilon$. The Killing spinor equations of type-IIB are

$$
\begin{aligned}
& \delta \lambda=\frac{1}{2} \not \supset \epsilon-\frac{1}{24} \not H \sigma_{3} \epsilon+\frac{1}{2} e^{\Phi}\left[\not F_{1}\left(i \sigma^{2}\right)+\frac{1}{12} \not_{3} \sigma^{1}\right] \epsilon, \\
& \delta \psi_{\mu}=D_{\mu} \epsilon-\frac{1}{8} H_{\mu \nu \rho} \Gamma^{\nu \rho} \sigma^{3} \epsilon-\frac{e^{\Phi}}{8}\left[\not F_{1}\left(i \sigma^{2}\right)+\frac{1}{6} \not F_{3} \sigma^{1}+\frac{1}{240} \not F_{5}\left(i \sigma^{2}\right)\right] \Gamma_{\mu} \epsilon,(
\end{aligned}
$$

where as always we are using the notation $\ddot{F}_{n} \equiv F_{i_{1} \ldots i_{n}} \Gamma^{i_{1} \ldots i_{n}}$.

\section{B Details of various KK reductions}

\section{B.1 Reduction of type-IIB on $S^{3}$}

In performing the reduction on $S^{3}$ we first note that the type-IIB Bianchi identities (2.4) imply that

$$
\begin{aligned}
& d G_{1}=0, \quad d G_{3}=H \wedge G_{1}, \\
& d G_{2}=-m H, \quad d\left(e^{-3 A} \star_{7} G_{2}\right)+H \wedge G_{3}=0 .
\end{aligned}
$$

The first three relations may be integrated to give the field content (2.5). Similarly the type-IIB flux equations of motion (A.6) imply that

$$
\begin{aligned}
& d\left(e^{3 A-2 \Phi} \star_{7} H\right)-e^{3 A} G_{1} \wedge \star_{7} G_{3}-G_{3} \wedge G_{2}+m e^{-3 A} \star_{7} G_{2}=0, \\
& d\left(e^{3 A} \star_{7} G_{1}\right)+e^{3 A} H \wedge \star_{7} G_{3}=0, \\
& d\left(e^{3 A} \star_{7} G_{3}\right)+H \wedge G_{2}=0 .
\end{aligned}
$$

We are after an effective seven-dimensional action which can capture this reduction procedure. Variations of this action with respect to these potentials will give equations 
of the form $d\left(\ldots \star_{7} G_{1,2,3}\right)=\cdots$. This implies that the last of (B.1) will arise from this action upon varying with respect to $C_{1}$.

The Einstein equations (A.4) reduce to a single constraint equation along $S^{3}$

$$
\frac{e^{-2 A}}{2}-3(\partial A)^{2}-D^{2} A+2 \partial A \cdot \partial \Phi=\frac{e^{2 \Phi}}{4}\left[m^{2} e^{-6 A}-G_{1}^{2}+\frac{1}{2} e^{-6 A} G_{2}^{2}-\frac{1}{6} G_{3}^{2}\right],
$$

where we have adopted the same normalisation as [4], namely $R_{m n}=\frac{1}{2} g_{m n}$, and the seven dimensional Einstein equation 12

$$
\begin{aligned}
R_{\mu v}= & -2 D_{\mu} D_{v} \Phi+3 D_{\mu} D_{v} A+3 D_{\mu} A D_{v} A+\frac{1}{4}(H)_{\mu \nu}^{2} \\
& +\frac{1}{2} e^{2 \Phi}\left[\left(G_{1}\right)_{\mu \nu}^{2}+e^{-6 A}\left(G_{2}\right)_{\mu \nu}^{2}+\frac{1}{2}\left(G_{3}\right)_{\mu \nu}^{2}\right. \\
& \left.-\frac{1}{2} g_{\mu \nu}\left(m^{2} e^{-6 A}+G_{1}^{2}+\frac{1}{2} e^{-6 A} G_{2}^{2}+\frac{1}{3 !} G_{3}^{2}\right)\right] .
\end{aligned}
$$

The one remaining equation to be considered is the type-II supergravity dilaton equation (A.5). In terms of seven-dimensional fields it reads

$$
R-6 D^{2} A-12(\partial A)^{2}+\frac{3}{2} e^{-2 A}+4 D^{2} \Phi+12 \partial A \cdot \partial \Phi-4(\partial \Phi)^{2}-\frac{1}{12} H^{2}=0 .
$$

One can repackage these equations of motion in an action with Lagrangian density

$$
\begin{aligned}
\mathcal{L} & =e^{3 A-2 \Phi}\left(R+6(\partial A)^{2}+4(\partial \Phi)^{2}-12 \partial A \cdot \partial \Phi-\frac{1}{12} H^{2}\right) \\
& -\frac{1}{2}\left(m^{2} e^{-3 A}-3 e^{A-2 \Phi}+e^{3 A} G_{1}^{2}+\frac{e^{-3 A}}{2} G_{2}^{2}+\frac{e^{3 A}}{6} G_{3}^{2}\right) \\
& +G_{2} \wedge C_{2} \wedge H
\end{aligned}
$$

where the last line is a topological term. In deriving the expression for the action we have made use of (B.5). As a quick consistency check, one can confirm that the constraint equation from the Einstein equations (B.3) appears by varying the action with respect to the scalar $A$, while (B.5) appears from varying the dilaton. Naturally, (B.4) appears from varying the action with respect to the metric.

\footnotetext{
${ }^{12}$ One should make use of the identity $\frac{1}{96}\left(\star_{7} G_{2}\right)_{\mu \nu}^{2}=\frac{1}{4}\left(G_{2}\right)_{\mu \nu}^{2}-\frac{1}{8} g_{\mu \nu} G_{2}^{2}$.
} 
We can now perform the conformal transformation

$$
g_{\mu v}=e^{\frac{4 \Phi-6 A}{5}} \hat{g}_{\mu v}
$$

resulting in the Einstein frame action with Lagrangian density

$$
\begin{aligned}
\mathcal{L}_{\text {Einstein }} & =R-\frac{24}{5}(\partial A)^{2}-\frac{4}{5}(\partial \Phi)^{2}+\frac{12}{5} \partial A \cdot \partial \Phi-\frac{1}{12} e^{\frac{12}{5} A-\frac{8}{5} \Phi} H^{2} \\
- & \frac{1}{2}\left(m^{2} e^{-\frac{36}{5} A+\frac{14}{5} \Phi}-3 e^{-\frac{16}{5} A+\frac{4}{5} \Phi}+e^{2 \Phi} G_{1}^{2}+\frac{1}{2} e^{-\frac{24}{5} A+\frac{6}{5} \Phi} G_{2}^{2}+\frac{1}{3 !} e^{\frac{12}{5} A+\frac{2}{5} \Phi} G_{3}^{2}\right) \\
+G_{2} & \wedge C_{2} \wedge H
\end{aligned}
$$

where the last line is a topological term and where we have dropped the hats.

\section{B.2 Reduction of massive IIA on the non-Abelian T-dual}

Here we present some details on the steps involved in this reduction. The metric (2.2) is of the form

$$
d s_{10}^{2}=d s^{2}\left(M_{7}\right)+f_{1}^{2} d r^{2}+f_{2}^{2} d s^{2}\left(S^{2}\right),
$$

where $f_{1}$ and $f_{2}$ depend on the coordinates of $M_{7}$ and in addition $f_{2}$ depends on $r$ as well. The result for the Ricci scalar is

$$
\begin{aligned}
\hat{R}= & R-2 \nabla^{2} \ln f_{1}-4 \nabla^{2} \ln f_{2}-2\left(\partial \ln f_{1}\right)^{2}-6\left(\partial \ln f_{2}\right)^{2}-4\left(\partial \ln f_{1}\right) \cdot\left(\partial \ln f_{2}\right) \\
& -\frac{2}{f_{1}^{2}}\left\{2 \partial_{r}^{2} \ln f_{2}+3\left(\partial_{r} \ln f_{2}\right)^{2}\right\}+\frac{2}{f_{2}^{2}} .
\end{aligned}
$$

If we substitute the expressions for $f_{1}, f_{2}$ as read off from (2.2), we obtain

$$
\begin{aligned}
\hat{R}= & R+\frac{4 r^{2} e^{4 A}+6 e^{8 A}-2 r^{4}}{\left(r^{2}+e^{4 A}\right)^{2}} D^{2} A+\frac{40 r^{2} e^{4 A}-12 e^{8 A}-4 r^{4}}{\left(r^{2}+e^{4 A}\right)^{2}}(\partial A)^{2} \\
& +\frac{2 e^{-2 A}}{\left(r^{2}+e^{4 A}\right)^{2}}\left(r^{4}+3 e^{4 A} r^{2}+9 e^{8 A}\right) .
\end{aligned}
$$

The corresponding expressions for the dilaton and the NS three-form are

$$
(\partial \hat{\Phi})^{2}=(\partial \Phi)^{2}+\frac{\left(r^{2}+3 e^{4 A}\right)^{2}}{\left(r^{2}+e^{4 A}\right)^{2}}(\partial A)^{2}-2 \frac{r^{2}+3 e^{4 A}}{r^{2}+e^{4 A}} \partial A \cdot \partial \Phi+\frac{r^{2}}{\left(r^{2}+e^{4 A}\right)^{2}}
$$


and

$$
\frac{\hat{H}^{2}}{12}=\frac{H^{2}}{12}+\frac{8 r^{2} e^{4 A}}{\left(r^{2}+e^{4 A}\right)^{2}}(\partial A)^{2}+\frac{e^{-2 A}}{2} \frac{\left(r^{2}+3 e^{4 A}\right)^{2}}{\left(r^{2}+e^{4 A}\right)^{2}} .
$$

The RR flux terms are

$$
\begin{aligned}
& \frac{1}{2} \hat{F}_{2}^{2}=m^{2} r^{2} e^{-4 A}+r^{2} e^{2 A} G_{1}^{2}+\frac{1}{2} G_{2}^{2}, \\
& \frac{1}{4 !} \hat{F}_{4}^{2}=e^{6 A} G_{1}^{2}+\frac{r^{2} e^{-4 A}}{2 !} G_{2}^{2}+\frac{r^{2} e^{2 A}}{3 !} G_{3}^{2}+\frac{e^{6 A}}{4 !}\left(\star_{7} G_{3}\right)^{2} .
\end{aligned}
$$

We also note the identity $\left(\star_{7} G_{3}\right)^{2}=-4 G_{3}^{2}$. Finally, the topological term of the massive IIA theory becomes

$$
-\frac{1}{2} \int_{M_{11}} \hat{F}_{4} \wedge \hat{F}_{4} \wedge \hat{H}=\int_{M_{10}} \frac{r^{4}}{r^{2}+e^{4 A}} C_{2} \wedge G_{2} \wedge H \wedge d r \wedge \operatorname{Vol}\left(S^{2}\right) .
$$

\section{B.3 Reduction of massive IIA on $S^{3}$}

The Bianchi identities (A.9) of massive IIA supergravity imply that

$$
d G_{1}=0, \quad d G_{2}=m H, \quad d G_{4}=H \wedge G_{2},
$$

which can be integrated to give the field content (4.3). Similarly the massive IIA flux eqs. (A.13) imply that

$$
\begin{aligned}
& d\left(e^{3 A-2 \Phi} \star_{7} H\right)-e^{3 A} G_{2} \wedge \star_{7} G_{4}-G_{1} \wedge G_{4}=m e^{3 A} \star_{7} G_{2}, \\
& d\left(e^{3 A} \star_{7} G_{2}\right)+e^{3 A} H \wedge \star_{7} G_{4}=0, \\
& d\left(e^{3 A} \star_{7} G_{4}\right)+H \wedge G_{1}=0, \quad d\left(e^{-3 A} \star_{7} G_{1}\right)+H \wedge G_{4}=0 .
\end{aligned}
$$

The Einstein equations reduce to

$$
\frac{1}{2} e^{-2 A}-3(\partial A)^{2}+2 \partial A \cdot \partial \Phi-D^{2} A=-\frac{e^{2 \Phi}}{4}\left(m^{2}-e^{-6 A} G_{1}^{2}+\frac{1}{2} G_{2}^{2}+\frac{1}{4 !} G_{4}^{2}\right)
$$

and the seven-dimensional Einstein equations

$$
\begin{aligned}
R_{\mu \nu}-3 \partial_{\mu} A \partial_{\nu} A-3 D_{\mu} D_{v} A+2 D_{\mu} D_{\nu} \Phi-\frac{1}{4}\left(H^{2}\right)_{\mu v} \\
=\frac{e^{2 \Phi-3 A}}{2}\left\{e^{-3 A} G_{1 \mu} G_{1 v}+e^{3 A}\left(G_{2}^{2}\right)_{\mu v}+\frac{e^{3 A}}{3 !}\left(G_{4}^{2}\right)_{\mu v}\right.
\end{aligned}
$$




$$
\left.-\frac{g_{\mu v}}{2}\left(e^{3 A} m^{2}+e^{-3 A} G_{1}^{2}+\frac{e^{3 A}}{2} G_{2}^{2}+\frac{e^{3 A}}{4 !} G_{4}^{2}\right)\right\} .
$$

The dilaton equation is the same as in (B.5).

The equations above can be obtained from an action with Lagrangian density

$$
\begin{aligned}
\mathcal{L}= & e^{3 A-2 \Phi}\left(R+6(\partial A)^{2}-12 \partial \Phi \cdot \partial A+4(\partial \Phi)^{2}-\frac{H^{2}}{12}+\frac{3}{2} e^{-2 A}\right) \\
& -\frac{1}{2}\left(m^{2} e^{3 A}+e^{-3 A} G_{1}^{2}+\frac{e^{3 A}}{2} G_{2}^{2}+\frac{e^{3 A}}{4 !} G_{4}^{2}\right) \\
& -G_{4} \wedge G_{1} \wedge B+\frac{m}{3} G_{1} \wedge B^{3}+\frac{1}{2} B^{2} \wedge d C_{1} \wedge G_{1}
\end{aligned}
$$

where the last line is a topological term. Passing to the Einstein frame using (B.7) we obtain

$$
\begin{aligned}
& \mathcal{L}_{\text {Einstein }}=R-\frac{24}{5}(\partial A)^{2}-\frac{4}{5}(\partial \Phi)^{2}+\frac{12}{5} \partial A \cdot \partial \Phi-\frac{1}{12} e^{\frac{12}{5} A-\frac{8}{5} \Phi} H^{2} \\
& -\frac{1}{2}\left(m^{2} e^{\frac{14}{5} \Phi-\frac{6}{5} A}-3 e^{\frac{4}{5} \Phi-\frac{16}{5} A}+e^{-6 A+2 \Phi} G_{1}^{2}+\frac{1}{2} e^{\frac{6}{5} A+\frac{6}{5} \Phi} G_{2}^{2}+\frac{1}{4 !} e^{\frac{18}{5} A-\frac{2}{5} \Phi} G_{4}^{2}\right) \\
& -G_{4} \wedge G_{1} \wedge B+\frac{m}{3} G_{1} \wedge B^{3}+\frac{1}{2} B^{2} \wedge d C_{1} \wedge G_{1} .
\end{aligned}
$$

\section{Details of derivation of the T-dual Ramond fields}

In computing the non-Abelian dual Ramond fields we need the Hodge duals as well, since in the definition of the bispinors (2.10) and (2.15) we use the democratic formulation in which all forms of degree up to ten appear [58]. The right degrees of freedom appear by impossing the constraint

$$
F_{p}=(-1)^{\left[\frac{p}{2}\right]} \star F_{10-p},
$$

valid in Minkowskian signature spacetimes. 


\section{C.1 Type-IIB to massive IIA}

The Hodge duals of the RR fluxes (2.4) are

$$
\begin{gathered}
F_{7}=-\left(\star F_{3}\right)=-m e^{-3 A} \operatorname{Vol}\left(M_{7}\right)-e^{3 A} \star_{7} G_{3} \wedge \operatorname{Vol}\left(S^{3}\right) \\
=-m e^{-3 A} e^{0} \wedge e^{1} \wedge \cdots \wedge e^{6}-\star_{7} G_{3} \wedge e^{7} \wedge e^{8} \wedge e^{9}, \\
F_{9}=\star F_{1}=e^{3 A} \star_{7} G_{1} \wedge \operatorname{Vol}\left(S^{3}\right)=\star_{7} G_{1} \wedge e^{7} \wedge e^{8} \wedge e^{9} .
\end{gathered}
$$

Also

$$
\star H=e^{3 A} \star_{7} H \wedge \operatorname{Vol}\left(S^{3}\right) .
$$

To present the T-dual RR fluxes we define the forms

$$
\begin{aligned}
& L_{1}=\mathbf{x} \cdot \hat{\mathbf{e}}=e^{-A} r d r, \\
& L_{2}=x_{7} \hat{e}^{8} \wedge \hat{e}^{9}+(\operatorname{cyclic} \text { in } 7,8,9)=e^{2 A} \tilde{B}=\frac{e^{2 A} r^{3}}{r^{2}+e^{4 A}} \operatorname{Vol}\left(S^{2}\right), \\
& L_{3}=\hat{e}^{7} \wedge \hat{e}^{8} \wedge \hat{e}^{9}=\frac{r^{2} e^{A}}{r^{2}+e^{4 A}} d r \wedge \operatorname{Vol}\left(S^{2}\right),
\end{aligned}
$$

where in the last step we used (2.13) and spherical coordinates. They obey the identities

$$
L_{1} \wedge L_{2}=r^{2} L_{3}, \quad L_{1}=\star_{3} L_{2}, \quad L_{2}=\star_{3} L_{1}
$$

Using the transformation

$$
\hat{P}=P \Omega^{-1}
$$

with $\Omega$ as in (2.12) we arrive after some algebra at

$$
\begin{aligned}
& \hat{F}_{0}=m, \\
& \hat{F}_{2}=-G_{2}+m \tilde{B}-e^{A} G_{1} \wedge L_{1}, \\
& \hat{F}_{4}=e^{3 A} \star_{7} G_{3}-e^{A} G_{3} \wedge L_{1}-G_{2} \wedge \tilde{B}+e^{3 A} G_{1} \wedge L_{3}, \\
& \hat{F}_{6}=e^{3 A} G_{3} \wedge L_{3}+e^{3 A} \star_{7} G_{3} \wedge \tilde{B}+e^{-2 A} \star_{7} G_{2} \wedge L_{1}-e^{3 A} \star_{7} G_{1}, \\
& \hat{F}_{8}=m e^{-2 A} \operatorname{Vol}\left(M_{7}\right) \wedge L_{1}-\star_{7} G_{2} \wedge L_{3}-e^{A} \star_{7} G_{1} \wedge L_{2}, \\
& \hat{F}_{10}=-m \operatorname{Vol}\left(M_{7}\right) \wedge L_{3}=-m \operatorname{Vol}\left(M_{10}\right) .
\end{aligned}
$$

One may check that the Bianchi identities and the flux equations of massive IIA are 
indeed obeyed using the corresponding formulae for type-IIB. In doing so the identity

$$
d\left(e^{3 A} L_{3}\right)+e^{A} \tilde{H} \wedge L_{1}=0,
$$

where $\tilde{H}=d \tilde{B}$, proves useful. One may also check that (C.1) is obeyed and therefore we may use $F_{p}$, with $p=0,2,4$ as the independent flux forms.

\section{C.2 Massive IIA to IIB}

In this case the Hodge duals of the RR fluxes (4.2) are

$$
\begin{aligned}
& F_{6}=-\left(\star F_{4}\right)=-e^{3 A} \star_{7} G_{4} \wedge \operatorname{Vol}\left(S^{3}\right)-e^{-3 A} \star_{7} G_{1}, \\
& F_{8}=\star F_{2}=e^{3 A} \star_{7} G_{2} \wedge \operatorname{Vol}\left(S^{3}\right), \\
& F_{10}=-\left(\star F_{0}\right)=-m e^{3 A} \operatorname{Vol}\left(M_{7}\right) \wedge \operatorname{Vol}\left(S^{3}\right) .
\end{aligned}
$$

Using ([C.6) we arrive after some algebra at

$$
\begin{aligned}
& \hat{F}_{1}=-G_{1}-m e^{A} L_{1}, \\
& \hat{F}_{3}=e^{3 A} \star_{7} G_{4}-e^{A} G_{2} \wedge L_{1}-G_{1} \wedge \tilde{B}+m e^{3 A} L_{3}, \\
& \hat{F}_{5}=e^{3 A} \star_{7} G_{4} \wedge \tilde{B}+e^{3 A} G_{2} \wedge L_{3}-e^{3 A} \star_{7} G_{2}-e^{A} G_{4} \wedge L_{1}, \\
& \hat{F}_{7}=e^{3 A} G_{4} \wedge L_{3}+e^{-2 A} \star_{7} G_{1} \wedge L_{1}-e^{3 A} \star_{7} G_{2} \wedge \tilde{B}+m e^{3 A} \operatorname{Vol}\left(M_{7}\right), \\
& \hat{F}_{9}=m e^{A} \operatorname{Vol}\left(M_{7}\right) \wedge L_{2}-\star_{7} G_{1} \wedge L_{3} .
\end{aligned}
$$

One may also check that the Bianchi identities and the flux equations of type-IIB are indeed obeyed using the corresponding formulae for massive IIA. Furthermore, (C.1) is obeyed and therefore we may use $F_{p}$, with $p=1,3,5$ as the independent flux forms.

\section{References}

[1] B.E. Fridling and A. Jevicki, Dual representations and ultraviolet divergences in nonlinear sigma models, Phys. Lett. B134 (1984) 70.

[2] E.S. Fradkin and A.A. Tseytlin, Quantum equivalence of dual field theories, Ann. Phys. 162 (1985) 31. 
[3] X.C. de la Ossa and F. Quevedo, Duality symmetries from non abelian isometries in string theory, Nucl. Phys. B403 (1993) 377, arXiv: hep-th/9210021.

[4] K. Sfetsos and D.C. Thompson, On non-abelian T-dual geometries with Ramond fluxes, Nucl. Phys. B846 (2011) 21, arXiv:1012.1320 [hep-th],

[5] Y. Lozano, E. O. Colgain, K. Sfetsos and D. C. Thompson, Non-abelian T-duality, Ramond fields and coset geometries, JHEP 1106 (2011) 106, arXiv:1104.5196 [hep-th].

[6] E. Bergshoeff, C. M. Hull and T. Ortin, Duality in the type II superstring effective action, Nucl. Phys. B451 (1995) 547, arXiv: hep-th/9504081.

[7] H. Samtleben and M. Weidner, The maximal $D=7$ supergravities, Nucl. Phys. B725 (2005) 383, arXiv: hep-th/0506237.

[8] M. Cvetic et al., Embedding AdS black holes in ten and eleven dimensions, Nucl. Phys. B558 (1999) 96, arXiv:hep-th/9903214.

[9] H. Lu, C.N. Pope and T.A. Tran, Five-dimensional $N=4, S U(2) \times U(1)$ gauged supergravity from type-IIB, Phys. Lett. B475 (2000) 261, arXiv:hep-th/9909203.

[10] M. Cvetic, H. Lu, C.N. Pope, A. Sadrzadeh and T. A. Tran, Consistent SO(6) reduction of type-IIB supergravity on $S^{5}$, Nucl. Phys. B586 (2000) 275, arXiv:hep-th/0003103.

[11] A. Khavaev, K. Pilch and N. P. Warner, New vacua of gauged $N=8$ supergravity in five dimensions, Phys. Lett. B487 (2000) 14, arXiv: hep-th/9812035.

[12] B. de Wit and H. Nicolai, The consistency of the $S^{7}$ truncation in $D=11$ supergravity, Nucl. Phys. B281 (1987) 211.

[13] H. Nastase, D. Vaman and P. van Nieuwenhuizen, Consistent nonlinear KK reduction of 11d supergravity on AdS(7) $x S(4)$ and self-duality in odd dimensions, Phys. Lett. B469 (1999) 96, arXiv: hep-th/9905075;

H. Nastase, D. Vaman and P. van Nieuwenhuizen, Consistency of the $A d S_{7} \times S^{4}$ reduction and the origin of self-duality in odd dimensions, Nucl. Phys. B581 (2000) 179, arXiv:hep-th/9911238. 
[14] H. Nicolai and K. Pilch, Consistent truncation of $d=11$ supergravity on $A d S_{4} \times S^{7}$, JHEP 1203 (2012) 099, arXiv:1112.6131 [hep-th].

[15] A. -K. Kashani-Poor, Nearly Kaehler Reduction, JHEP 0711 (2007) 026, arXiv:0709.4482 [hep-th].

[16] D. Cassani and A. -K. Kashani-Poor, Exploiting $N=2$ in consistent coset reductions of type IIA, Nucl. Phys. B817 (2009) 25, arXiv:0901.4251] [hep-th].

[17] J. P. Gauntlett, S. Kim, O. Varela and D. Waldram, Consistent supersymmetric Kaluza-Klein truncations with massive modes, JHEP 0904 (2009) 102, arXiv:0901.0676 [hep-th].

[18] E. O Colgain, O. Varela and H. Yavartanoo, Non-relativistic M-Theory solutions based on Kaehler-Einstein spaces, JHEP 0907 (2009) 081, arXiv:0906.0261 [hep-th].

[19] E. O Colgain and H. Samtleben, 3D gauged supergravity from wrapped M5-branes with AdS/CMT applications, JHEP 1102 (2011) 031, arXiv:1012.2145 [hep-th].

[20] D. Cassani, G. Dall'Agata and A. F. Faedo, Type-IIB supergravity on squashed Sasaki-Einstein manifolds, JHEP 1005 (2010) 094, arXiv:1003.4283 [hep-th].

[21] K. Skenderis, M. Taylor and D. Tsimpis, A Consistent truncation of IIB supergravity on manifolds admitting a Sasaki-Einstein structure, JHEP 1006 (2010) 025, arXiv:1003.5657 [hep-th].

[22] J. P. Gauntlett and O. Varela, Universal Kaluza-Klein reductions of type-IIB to $N=4$ supergravity in five dimensions, JHEP 1006 (2010) 081, arXiv:1003.5642 [hep-th].

[23] J. T. Liu, P. Szepietowski and Z. Zhao, Consistent massive truncations of IIB supergravity on Sasaki-Einstein manifolds, Phys. Rev. D81 (2010) 124028, arXiv:1003.5374 [hep-th].

[24] D. Cassani and P. Koerber, Tri-Sasakian consistent reduction, JHEP 1201 (2012) 086, arXiv:1110.5327 [hep-th].

[25] I. Bah, A. Faraggi, J. I. Jottar, R. G. Leigh and L. A. Pando Zayas, Fermions and $D=11$ supergravity on squashed Sasaki-Einstein manifolds, JHEP 1102 (2011) 068, arXiv:1008.1423 [hep-th]; 
I. Bah, A. Faraggi, J. I. Jottar and R. G. Leigh, Fermions and type-IIB supergravity on squashed Sasaki-Einstein manifolds, JHEP 1101 (2011) 100, arXiv:1009.1615 [hep-th].

[26] M. Cvetic, H. Lu, C. N. Pope, A. Sadrzadeh and T. A. Tran, $S^{3}$ and $S^{4}$ reductions of type-IIA supergravity, Nucl. Phys. B590 (2000) 233, arXiv: hep-th/0005137.

[27] E. Ó Colgáin and O. Varela, Consistent reductions from $D=11$ beyond Sasaki-Einstein, Phys. Lett. B703 (2011) 180, arXiv:1106.4781 [hep-th],

[28] K. Sfetsos, NonAbelian duality, parafermions and supersymmetry, Phys. Rev. D54 (1996) 1682, arXiv: hep-th/9602179.

[29] S.F. Hassan, T-duality, space-time spinors and R-R fields in curved backgrounds, Nucl. Phys. B568 (2000) 145, arXiv: hep-th/9907152.

[30] M. Blau, J. M. Figueroa-O'Farrill, C. Hull and G. Papadopoulos, A New maximally supersymmetric background of IIB superstring theory, JHEP 0201 (2002) 047, arXiv: hep-th/0110242.

[31] H. Lin, O. Lunin and J. M. Maldacena, Bubbling AdS space and 1/2 BPS geometries, JHEP 0410 (2004) 025, arXiv : hep-th/0409174.

[32] K. Balasubramanian and K. Narayan, Lifshitz spacetimes from AdS null and cosmological solutions, JHEP 1008 (2010) 014, arXiv:1005.3291 [hep-th].

[33] C. Klimcik and P. Severa, Poisson-Lie T duality and loop groups of Drinfeld doubles, Phys. Lett. B372 (1996) 65, arXiv: hep-th/9512040.

[34] A. H. Chamseddine and W. A. Sabra, $D=7$ SU(2) gauged supergravity from $D=10$ supergravity, Phys. Lett. B476 (2000) 415 , arXiv: hep-th/9911180.

[35] P. K. Townsend and P. van Nieuwenhuizen, Gauged seven-dimensional supergravity, Phys. Lett. B125 (1983) 41.

[36] M. Cvetic, H. Lu and C. N. Pope, Consistent Kaluza-Klein sphere reductions, Phys. Rev. D62 (2000) 064028, arXiv: hep-th/0003286.

[37] A. Salam and E. Sezgin, SO(4) gauging of $N=2$ supergravity in seven-dimensions, Phys. Lett. B126 (1983) 295. 
[38] J. F. Morales and H. Samtleben, Higher spin holography for SYM in d dimensions, Phys. Lett. B607 (2005) 286, arXiv: hep-th/0411246

[39] L. J. Romans, Gauged N=4 supergravities in five-dimensions and their magnetovac backgrounds, Nucl. Phys. B267 (1986) 433.

[40] M. Cvetic, H. Lu and C. N. Pope, Consistent warped-space Kaluza-Klein reductions, half-maximal gauged supergravities and CP(n) constructions, Nucl. Phys. B597 (2001) 172, arXiv:hep-th/0007109.

[41] J. P. Gauntlett and O. Varela, $D=5 S U(2) x U(1)$ gauged supergravity from $D=11$ supergravity, JHEP 0802 (2008) 083, arXiv:0712.3560 [hep-th].

[42] E. Alvarez, L. Alvarez-Gaume, J.L.F. Barbon and Y. Lozano, Some global aspects of duality in string theory, Nucl. Phys. B415 (1994) 71, arXiv:hep-th/9309039.

[43] A.P. Polychronakos and K. Sfetsos, High spin limits and non-abelian T-duality, Nucl. Phys. B843 (2011) 344, arXiv:1008.3909 [hep-th].

[44] Y. Kosmann, A note on Lie-Lorentz derivatives, Annali di Mat. Pura Appl. (IV) 91 (1972) 317.

[45] J. M. Figueroa-O'Farrill, On the supersymmetries of Anti-de Sitter vacua, Class. Quant. Grav. 16 (1999) 2043, arXiv: hep-th/9902066.

[46] T. Ortin, A note on Lie-Lorentz derivatives, Class. Quant. Grav. 19 (2002) L143, arXiv:hep-th/0206159.

[47] A. Buchel, Coarse-graining 1/2 BPS geometries of type IIB supergravity, Int. J. Mod. Phys. A21 (2006) 3495, arXiv: hep-th/0409271.

[48] M. M. Caldarelli, D. Klemm and P. J. Silva, Chronology protection in anti-de Sitter, Class. Quant. Grav. 22 (2005) 3461, arXiv: hep-th/0411203.

[49] I. Bena and N. P. Warner, A Harmonic family of dielectric flow solutions with maximal supersymmetry, JHEP 0412 (2004) 021, arXiv: hep-th/0406145.

[50] S. Kachru, X. Liu and M. Mulligan, Gravity duals of Lifshitz-like fixed points, Phys. Rev. D78 (2008) 106005, arXiv:0808.1725 [hep-th]. 
[51] A. Donos and J. P. Gauntlett, Lifshitz solutions of $D=10$ and $D=11$ supergravity, JHEP 1012 (2010) 002, arXiv: 1008.2062 [hep-th],

[52] P. Candelas and X. C. de la Ossa, Comments on conifolds, Nucl. Phys. B342 (1990) 246.

[53] J. P. Gauntlett, D. Martelli, J. Sparks and D. Waldram, Sasaki-Einstein metrics on $S^{2} \times S^{3}$, Adv. Theor. Math. Phys. 8 (2004) 711, arXiv: hep-th/0403002.

[54] S. R. Das, J. Michelson, K. Narayan and S. P. Trivedi, Time dependent cosmologies and their duals, Phys. Rev. D74 (2006) 026002, arXiv: hep-th/0602107.

[55] C.P. Burgess, N.E. Grandi, F. Quevedo and R. Rabadan, D-brane chemistry, JHEP 01 (2004) 067, arXiv: hep-th/0310010.

[56] K.-M. Lee, E. O Colgain, H. Yavartanoo and K.P. Yogendran, BPS D0-D6 Branes in supergravity, JHEP 0903 (2009) 009, arXiv:0811.2086 [hep-th],

[57] J. Maldacena and J.R. Russo, Large N limit of non-commutative gauge theories, JHEP 09 (1999) 025, arXiv: hep-th/9908134.

[58] E. Bergshoeff, R. Kallosh, T. Ortin, D. Roest and A. Van Proeyen, New formulations of $D=10$ supersymmetry and D8-O8 domain walls, Class. Quant. Grav. 18 (2001) 3359, arXiv: hep-th/0103233.

[59] D. Gaiotto, J. Maldacena, The gravity duals of $N=2$ superconformal field theories, arXiv:0904.4466 [hep-th]. 